\title{
Consumo de bebidas alcohólicas en estudiantes universitarios: motivaciones y estilos de afrontamiento
}

\section{Cecilia Chau}

Universidad de Lima

Lima, Perú

Este artículo evalúa las relaciones entre el consumo de bebidas alcohólicas, la tipología del bebedor, las motivaciones para beber y los estilos de afrontamiento en un grupo de universitarios de uno y otro género, de los primeros años de estudios de una universidad privada en Lima. Los resultados confirman que el uso de bebidas alcohólicas es frecuente y moderado en la mayoría de estos jóvenes (55\%), sobre todo en los hombres. Sin embargo, se encuentra un grupo de bebedores problemáticos importante (22\%) que no debe dejarse de lado. Asimismo, los jóvenes que más beben reportan mayores problemas referidos a su consumo. Los motivos para beber se convierten en un elemento relevante respecto a la cantidad de alcohol consumido y los problemas relacionados.

tipología del bebedor / motivaciones para beber / estilos de afrontamiento / bebida alcohólica

\section{Alcoholic beverage consumption in college students: motivations and coping styles}

The relationship between consumption of alcoholic beverages, the typology of the drinker, motivation for drinking, and coping styles in a group of both male and female university students of the first years of study in a private university in Lima, were evaluated. The results confirm that the use of alcoholic beverages is frequent and moderate among the majority of these young people (55\%), especially among men. Nevertheless, we find a significant group of problematic drinkers (22\%) that should not be ignored. Likewise, the youngsters who drink more report major problems related to their consumption. The motivations for drinking become a relevant element with respect to the amount of alcohol consumed and related problems.

typology of drinkers / motivations for drinking / coping styles /alcoholic beverage

Dirección de la autora: Chau@correo.ulima.edu.pe 
Durante los últimos veinte años se ha registrado en los países desarrollados un alto grado de preocupación por el aumento de la disponibilidad de diversas sustancias psicoactivas y el incremento de los problemas sanitarios y legales derivados de su consumo. Sin embargo, los profesionales vinculados al estudio de las conductas adictivas, consideran que es absolutamente necesario volver a prestar atención a la problemática generada por el alcohol.

El consumo excesivo de bebidas alcohólicas en la población universitaria ha sido objeto de diversos estudios en prácticamente todos los continentes. Tales investigaciones comenzaron a mediados de los años setenta en los países industrializados, posteriormente, a partir de 1980, diferentes estudios revelaron los altos niveles de consumo y los problemas asociados a su abuso en los estudiantes universitarios (O'Coneell \& Patterson, 1989).

Las investigaciones sobre el consumo de sustancias psicoactivas, incluido el alcohol, llevadas a cabo en nuestro medio, y que abarcan la población total, comenzaron a realizarse a finales de la década de los setenta, sin diferenciar grupos específicos.

En los últimos estudios realizados por CEDRO (Centro de Información y Educación para la Prevención del Abuso de Drogas) $(1993,1997)$ se observa un patrón de consumo que se caracteriza por el uso generalizado de bebidas alcohólicas y, en segundo término, del tabaco; y, según Saavedra (1997), en el Perú el alcoholismo constituye la principal dependencia a sustancias psicoactivas, la que produce los mayores estragos, tanto a nivel personal y familiar, como social y comunitario.

Cabe señalar que las investigaciones sobre el fenómeno de uso de drogas en el Perú son principalmente de corte epidemiológico (Jutkowitz, Arellano, Castro De La Mata, Davis, Elinson, Jerí, Shaycoft y Timaná, 1987; Ferrando, 1990, 1991; CEDRO, 1993, 1997), realizadas en una población general de 12 a 50 años. Además, se han hecho estudios en población escolar (Sotomayor, 1976; Huamán, Tueros y Villanueva, 1983; León, Ugarriza y Villanueva, 1989; Villanueva, 1989a; Ferrando, 1992; Villanueva, 1996).

Sin embargo, se han realizado pocos trabajos en grupos específicos, como los universitarios. En este sentido, Ferrando (1991) se aproxima a la edad comprendida por quienes cursan una carrera universitaria, al investigar a jóvenes de 17 a 24 años, encontrando que la prevalencia de vida del alcohol (uso de una sustancia alguna vez en la vida de una persona), entre adolescentes de 15 y 17 años era de $61 \%$, y $89 \%$ entre los de 21 y 24 años.

Los aspectos psicológicos relacionados al consumo de bebidas alcohólicas entre los universitarios como grupo específico han sido poco estudiados. Es por ello que surgió el interés de investigar y conocer las características de es- 
tos jóvenes que entre los de 19 y 24 años reportan uso actual y reciente del alcohol en un $52,4 \%$, siendo mayor la prevalencia de vida del alcohol en los hombres que en las mujeres (CEDRO, 1997).

Si bien son muchos los factores que contribuyen a que una persona beba alcohol, se considera que el origen común a esta conducta es motivacional (Cox \& Klinger, 1988). Por otro lado, el modelo de afrontamiento señala que ciertos tipos de "esfuerzos conductuales y cognitivos que hace el individuo para hacer frente al estrés" (Lazarus y Folkman, 1986), podrían disminuir o incrementar el riesgo de abuso del alcohol, cuando otros factores de vulnerabilidad están presentes.

\section{TIPOLOGÍA DEL BEBEDOR}

La medición de conductas relacionadas con el alcohol entre los adolescentes comparte algunas similitudes con la de los adultos, así como también muestra algunas diferencias. Por ejemplo, al igual que en la literatura sobre adultos, las investigaciones recientes sobre adolescentes se han centrado en la heterogeneidad de las conductas relacionadas a la bebida, que incluyen no sólo el consumo en sí mismo, sino también las consecuencias sociales adversas y los síntomas de dependencia al alcohol. Estos modelos multidimensionales de las conductas del adolescente relacionadas con el alcohol han dado lugar de dos a seis dimensiones, y tres de ellas son relativamente invariables en todos los estudios. Estas tres dimensiones se refieren a la intensidad del consumo de alcohol, a las consecuencias negativas o adversas, y a los síntomas de dependencia, tales como la pérdida de control (Windle, 1996).

Windle en 1996 propuso una tipología del bebedor para adolescentes que está influida por los enfoques epidemiológicos del alcohol en adultos, con respecto a la gradación continua en la conducta de beber, incluidos los problemas con la bebida. Se aleja de la conceptualización clásica dicotómica de los criterios diagnósticos.

Se propone que esta aproximación en la medición centrada en la gradación continua podría ser más sensible en la conducta de beber en los adolescentes, que los sistemas diagnósticos estándares o los enfoques unidimensionales (alcohol consumido). El enfoque tipológico propuesto podría captar de forma más adecuada las sutiles variaciones en el involucramiento con el alcohol entre los adolescentes que otras aproximaciones. Estas variaciones podrían a su vez reflejar vías etiológicamente distintas, identificar riesgos diferenciales para el desarrollo del alcoholismo en el adulto, y sugerir medidas de prevención e intervenciones alternativas para distintas subpoblaciones de adolescentes. Esta propuesta es primordial, si se considera la limitada eficacia de los esfuerzos preventivos actuales desarrollados con los adolescentes, en los cuales se ha re- 
currido mayoritariamente a intervenciones uniformes, en lugar de considerar el grado de involucramiento con el alcohol.

El Instituto de Medicina de Estados Unidos en 1990 (Windle, 1996) reportó que la proporción de adultos bebedores-problema, en relación con los dependientes del alcohol, era aproximadamente de 4 a 1 . Se estima que esta proporción es mayor entre los adolescentes. El bebedor problemático ha sido definido como el individuo que abusa del alcohol, pero que no es alcohólico (Madrigal, 1998). Desde la perspectiva clínica, Windle (1996) plantea que una distinción más importante debe hacerse entre los adolescentes cuyo consumo es intenso y problemático. A pesar de ser discutible, esta distinción se ubica como fundamental para distinguir entre aquéllos que, al menos actualmente, parecen ser capaces de consumir cantidades relativamente grandes de alcohol, con pocas consecuencias adversas, y aquéllos que consumen también grandes cantidades de alcohol, pero tienen un gran número de consecuencias adversas.

Desde el punto de vista clínico, la tipología de Windle presenta ventajas: entre otras, permite centrarse en posibles diferencias entre los bebedores problema y los bebedores intensos, distinción que podría ser útil para identificar a aquéllos que necesitan intervención intensiva y extensiva; y ofrece características identificables que permi- ten definir un inicio temprano, incremento y mantenimiento de un consumo problemático. La tipología también podría ser utilizada para ubicar adolescentes bebedores moderados e intensos que si bien, actualmente, no manifiestan consecuencias negativas relacionadas con el alcohol, podrían estar en riesgo de devenir en consumidores problemáticos.

Para evaluar las gradaciones con respecto a los niveles progresivos de involucramiento con el alcohol, Windle elaboró la tipología del bebedor, que cuenta con cinco categorías que abarcan desde el abstemio al problemático y su consumo en los últimos 30 días. El abstemio es aquél que no ha consumido un trago; el bebedor ligero es el que ha consumido al menos un trago pero menos de diez; el moderado es aquél que ha consumido diez o más tragos pero menos de 45; el bebedor intenso ha consumido más de 45 tragos o ha consumido seis o más tragos en al menos cada una de las tres últimas ocasiones en que consumió. Por último, el bebedor problemático es aquél que ha consumido más de 45 tragos o ha consumido seis o más tragos, en al menos cada una de las tres últimas ocasiones en que consumió y ha reportado cinco o más problemas con el alcohol en los últimos seis meses. Cada trago equivale a $350 \mathrm{ml}$ de cerveza (que es igual a una lata de cerveza o a 10-11 gr de etanol). 
El criterio utilizado para la categorización se basó en tres índices: a) el índice de la frecuencia/cantidad estándar que refleja el número promedio de onzas de etanol consumidas por día; b) el número de episodios de abuso (beber seis o más tragos en una ocasión) y c) el número de problemas con el alcohol (principalmente, consecuencias sociales adversas asociadas a su consumo). Los cortes para el índice de la frecuencia/cantidad y el número de los días de consumo intenso fueron consistentes con la literatura existente (OMS, 1994a).

Existen también los criterios planteados por The National Institute on Alcoholism and Alcohol Abuse (NIAA) (Schorling \& Buchsbaum, 1997), que recomienda no más de 14 tragos por semana en los hombres (el equivalente a ocho botellas grandes de $620 \mathrm{ml}$ de cerveza) y siete en las mujeres (o cuatro botellas grandes de $620 \mathrm{ml}$ de cerveza). Se define la categoría riesgo de consumo como el nivel de consumo que, al ser sobrepasado, puede conducir a consecuencias adversas sobre la salud pero que, en el tiempo presente, no genera suficientes efectos adversos que cubran los criterios de abuso o dependencia.

\section{MOTIVOS PARA CONSUMIR BEBIDAS ALCOHÓLICAS}

Cox y Klinger (1988) postularon un modelo motivacional del uso de alcohol en el que señalaron que el origen de esta conducta es motivacional, debido a que una persona decide consciente o inconscientemente consumir o no una bebida alcohólica, de acuerdo a si espera que las consecuencias afectivas positivas de beber tengan un mayor peso que aquéllas de no hacerlo. La motivación para beber está estrechamente ligada con los incentivos que tenga la persona en otras áreas de su vida, y a los cambios que ésta experimenta a raíz de ellos. Esto no quiere decir que los autores dejaron de reconocer que existen otros factores que favorecen el comportamiento de consumo de alcohol.

En el caso de la motivación para usar bebidas alcohólicas, ha sido demostrado que las expectativas acerca de los efectos del alcohol están presentes desde antes de que la persona lo consuma (Christiansen, Golman \& Inn, 1982). Éstas influyen dramáticamente en la motivación de las personas para hacerlo y en los efectos sobre su comportamiento.

Existen dos maneras en las que el uso del alcohol puede traer cambios afectivos y, con respecto a éstas, hay dos tipos de efectos correspondientes que las personas esperan obtener al beber. La primera es a través del efecto químico directo del alcohol sobre las emociones. Langenbucher y Natahn (Cox \& Klinger, 1988) señalaron que el alcohol tiene efectos sobre el humor, que generalmente es descrito como reducción de la tensión. En general, puede decirse que las personas consumen alcohol más por las expectativas que tienen sobre sus efectos (distensión), que por la ac- 
ción farmacológica del alcohol en sí (Marlatt \& Rohsenow, 1980). La segunda manera es indirecta. Ésta se produce cuando el alcohol juega un papel instrumental como incentivo en la interacción social de la persona para la obtención de una meta. Un incentivo se convierte en una meta cuando una persona se compromete a seguirlo, adquiriendo un estado motivacional distintivo (Klinger, 1975, Klinger, 1977). Por ejemplo, muchas variables sociales influyen indirectamente en el proceso de ingerir alcohol, ya que esta conducta es instrumental en el logro de la aceptación de las amistades.

Asimismo, beber alcohol, especialmente en cantidades excesivas, cambia el afecto de las personas, su motivación por incentivos y su posterior motivación para beber o no más alcohol. Lo anterior se entiende como la tendencia que tiene un organismo para seguir incentivos positivos por los cuales se siente atraído e incentivos negativos, que se rechazan.

Es así que Cox y Klinger (1988, 1990) propusieron una estructura para categorizar los motivos del consumo, a lo largo de dos dimensiones subyacentes que reflejan la valencia (positiva o negativa) y la fuente (interna o externa) de los resultados que un individuo espera obtener cuando bebe. Las personas podrían beber con el fin de obtener un resultado positivo (reforzamiento positivo) o evitar uno negativo (reforzamiento negativo). Incluso podría res- ponder a recompensas internas, tales como el manejo de los estados emocionales; o a recompensas externas, tales como la aceptación social y la aprobación. Al cruzar estas dos dimensiones se obtienen cuatro clases de motivos: a) generados internamente: los motivos de reforzamiento positivo (beber para estimularse, elevar el estado de ánimo o sentirse bien); b) generados externamente: los motivos de reforzamiento positivo (beber para obtener aceptación social); c) generados internamente: motivos de reforzamiento negativo (beber para reducir o regular las emociones negativas), y d) generados externamente: los motivos de reforzamiento negativo (beber para evitar la censura social o el rechazo).

En las investigaciones de los últimos años sobre los motivos relacionados con el consumo, los estudios se han focalizado sólo en dos de ellos: beber para regular el afecto negativo y beber para obtener recompensas sociales positivas (llamados motivos de afrontamiento y motivos sociales, respectivamente). Como consecuencia de ello, se conoce menos sobre el consumo de alcohol como una forma de estimularse, elevar el estado de ánimo, o para evitar costos sociales (llamados motivos de estimulación y motivos de conformidad).

Cox y Klinger en 1988 y Cooper en 1994 han reportado que los motivos sociales están más relacionados con el uso de alcohol ligero, infrecuente, no 
problemático y en situaciones sociales; mientras que los motivos de afrontamiento están más relacionados con características de consumo intenso, problemático y solitario. En estudios con adolescentes la evidencia sugiere que beber para elevar el estado de ánimo podría ser un importante factor motivacional a tomar en cuenta.

En relación con los motivos de conformidad, pocos adolescentes avalaron estas razones para su consumo, y esos motivos estuvieron poco relacionados con el uso frecuente o intenso de alcohol. Sin embargo, entre los adolescentes más jóvenes (12 a 14 años) estos motivos y el consumo de bebidas alcohólicas, podría ser predictivo de su uso entre ellos (Cox \& Klinger, 1988; Cooper, 1994).

Complementando los conceptos ya presentados, tenemos que Cooper et al. definieron en 1995 los motivos de afrontamiento para el uso de alcohol como maneras estratégicas de escapar, evadir o, de lo contrario, regular emociones negativas. Por el contrario, los motivos de estimulación implican el uso estratégico de alcohol para aumentar los estados afectivos positivos y se asume como un comportamiento emitido para lograr un estado deseado. Los motivos para consumir son vistos como determinantes del consumo.

A partir de este planteamiento se sugiere que las estrategias efectivas de intervención pueden diferir en función de los procesos motivacionales subyacen- tes. Así, por ejemplo, las intervenciones dirigidas a reducir los niveles de estrés y que ofrecen formas alternativas para su afrontamiento, pueden ser beneficiosas entre individuos que dependen del alcohol como una forma de enfrentarse a sus emociones negativas. En contraste, proporcionar fuentes alternativas de estimulación placentera o técnicas cognitivas de reestructuración (Beck, 1995) dirigidas a alterar las expectativas por los efectos reforzadores del alcohol, puede ser más apropiado para individuos que beben principalmente para aumentar la experiencia emocional.

\section{Afrontamiento en consumidores DE BEBIDAS ALCOHÓLICAS}

\section{Conceptualización del afrontamiento}

La definición clásica o teoría transaccional proviene de Lazarus y Folkman (1986), quienes entienden el afrontamiento como un proceso, una relación cambiante entre la persona y un contexto; es de carácter dinámico e irá variando según como se despliegue la situación. Lo plantean de la siguiente forma:

Definimos el afrontamiento como aquellos esfuerzos cognitivos y conductuales constantemente cambiantes que se desarrollan para manejar las demandas específicas externas y/o internas que son evaluadas como excedentes o desbordantes de los recursos del individuo (p. 164). 
Lazarus y Folkman inicialmente plantearon los tipos de afrontamiento, enfocados en la emoción y en el problema:

- Focalizarse en el problema. Se presenta cuando la persona busca cambiar la relación existente, sea alejando o disminuyendo el impacto de la amenaza.

- Focalizarse en la emoción. Está dirigido a disminuir o eliminar las reacciones emocionales desencadenadas por la fuente estresante. Asume que la situación tiene una condición estresante que es inmodificable y por ello centra toda su atención en disminuir el malestar emocional que se va a generar.

En los últimos años se ha ampliado esta posición sugiriéndose otros tipos:

- Estrategias diseñadas para cambiar el significado del estresor. Implican que van a controlar el significado de los eventos estresantes, neutralizando cognitivamente la evaluación de amenaza, pérdida o daño. Se plantean algunas estrategias:

- Realizar comparaciones positivas: se puede plantear que muchos otros experimentan esas situaciones de estrés.

- Ignorar selectivamente: tratar de olvidar el problema pues existen otros aspectos más importantes en la vida.

- Disminuir la importancia del evento (no magnificarlo).
- Hacer esfuerzos por crear significados positivos a un estresor (re-aprecio cognitivo).

\section{Estilos de afrontamiento y consumo de bebidas alcohólicas}

Las aproximaciones teóricas a la relación entre afrontamiento y uso de sustancias psicoactivas difiere de los modelos estrictamente conductuales, en los que se interpreta el uso de sustancias como una conducta aprendida, adquirida y mantenida en respuesta a las señales y presiones sociales; o de los modelos de desviación, los cuales interpretan el uso de dichas sustancias como una forma de conducta desviada motivada por el rechazo de los valores sociales convencionales y la adopción de un estilo de vida desviado (Jessor \& Jessor, 1977). Si bien los modelos de afrontamiento no rechazan esta evidencia, postulan la existencia de otros factores que predicen el incremento de riesgo en la persona.

Se presentan tres posiciones básicas acerca de las funciones del afrontamiento, en el uso de sustancias, derivadas del conocimiento de los efectos fisiológicos de su uso y de los estudios de los motivos percibidos para usar tabaco y alcohol (Pandina, Johnson \& Labovie, 1992).

La primera de ellas postula la regulación directa del afecto, esto es que la sustancia produce cambios en los estados afectivos. Por ejemplo, el modelo de reducción de la tensión por el uso de 
alcohol, postula que éste reduce la ansiedad. La segunda, postula que el uso de sustancias proporciona distracción de los problemas, a través de los procesos fisiológicos que operan desviando la atención, temporalmente, de la conciencia displacentera. La última sugiere que el uso de sustancias proporciona una mejor performancia a través de los efectos fisiológicos en el arousal u otros mecanismos.

La literatura de los modelos de las expectativas, especialmente los derivados del uso de alcohol, representan algunas combinaciones de estas funciones del afrontamiento. Uno de ellos es el modelo transaccional de Lazarus y Folkman (1986) y Lazarus (1991). Esta teoría asume implícitamente que los individuos tienen expectativas sobre la probable efectividad de las respuestas de afrontamiento en su repertorio de conductas. El modelo predice que ambos tipos de afrontamiento (centrado en el problema y en la emoción) son protectores, pues el afrontamiento centrado en el problema, en el caso del uso de sustancias psicoactivas, reduce el nivel de problemas que podría crear el estrés, y el afrontamiento centrado en la emoción reduce el nivel de malestar (distress) emocional interno.

\section{Procesos de afrontamiento: modelos de aproximación-evitación}

En este modelo la principal distinción es entre las respuestas que involucran inversión de esfuerzo al tratar con el problema versus respuestas en las cuales la persona no invierte esfuerzos (Carver et al., 1989; Endler \& Parker, 1990). Respuestas tales como afrontamiento focalizado en el problema (también llamado conductual o acción directa), afrontamiento cognitivo (también llamado evaluación) y afrontamiento social (búsqueda de soporte social) son todos agrupados bajo el modelo afrontamiento de aproximación, debido a que ellos involucran inversión activa de esfuerzo en el proceso de afrontamiento.

En contraste, se encuentra el afrontamiento evitativo que incluye respuestas tales como distracción, abandono, desentendimiento mental y conductual, enfocar y liberar emociones. En algunos estudios, el uso de drogas y medicación está explícitamente incluido en esta categoría, debido a que se agrupan empíricamente con otros mecanismos evitativos (Rohde, Lewinsohn, Tilson y Seeley, 1990).

Los resultados de estos modelos muestran en principio que los afrontamientos de aproximación son más adaptativos, mientras que los afrontamientos evitativos no lo son (Blechman \& Wills, 1992; Stone, Greenberg, Kennedy-Moore Newman, 1991). El supuesto implícito es que la mayor inversión de esfuerzo en el afrontamiento, conducirá a mejores resultados.

El modelo de afrontamiento del $a b u$ so de sustancias propuesto por Wills y Hirky en 1996 integra los conceptos 
antes discutidos. Asume que una persona puede incrementar el riesgo del uso de sustancias debido a factores de temperamento (alto nivel de actividad), factores sociales (un ambiente con pocos modelos adaptativos de habilidades de afrontamiento), o factores estresantes (muchos eventos de vida negativos). Estos factores antecedentes incrementan la vulnerabilidad debido a que elevan el malestar (distress) subjetivo, reducen el reforzamiento del ambiente social y hacen que el uso de sustancias se perciba como más atractivo. Estas funciones del afrontamiento incluyen regulación de los estados afectivos y distracción de la conciencia displacentera de la situación.

Lo interesante de este modelo es que postula que tipos particulares de afrontamiento pueden incrementar o disminuir el riesgo para el abuso de sustancias cuando los factores de vulnerabilidad están presentes. Los tipos de afrontamiento evitativo son predictivos en el incremento del riesgo debido a que el individuo no está dispuesto a tratar con las situaciones problemáticas, pero en lugar de ello busca caminos de menor resistencia hacia la restauración del balance afectivo. La persona tenderá a afrontar negando la existencia de los problemas y buscando distracción de la conciencia del displacer. En este caso, el uso de sustancias como mecanismo de afrontamiento podría ser más probable.

En contraste, los tipos activos de afrontamiento son predictivos de la disminución del riesgo. Una aproximación hacia la solución de problemas, es más probable que resuelva las situaciones problemáticas, incremente los sentimientos de autoeficacia y autoestima, y atraiga a otros a ayudar en el proceso de solución de problemas.

\section{Estilos de afrontamiento y uso de alcohol}

Los modelos teóricos del aprendizaje social conceptualizan el uso de alcohol como un método general para afrontar las demandas de cada día, que puede llegar a ser maladaptativo si es usado excesivamente (Evans \& Jo Dunn, 1995; Cooper, Russel, Skinner, Frone \& Mudar, 1992). Particularmente los individuos vulnerables son los que carecen de las habilidades de afrontamiento requeridas o la confianza para movilizar los recursos necesarios para ejercer control sobre las tareas demandadas. Además, la probabilidad de uso de alcohol se incrementa cuando un individuo mantiene expectativas positivas e intensas sobre los efectos del alcohol, tales como lograr resultados valiosos relativos a su consumo. A menos que respuestas más adaptativas de afrontamiento sean aprendidas, la dependencia al alcohol para afrontar las demandas situacionales probablemente continuará en el tiempo, y conducirá a dependencia física y psicológica (Marlatt, 1988; Evans \& Jo Dunn, 1995).

Muchos estudios sugieren que los estilos generales de afrontamiento pue- 
den moderar el impacto de los eventos estresantes de vida y el subsecuente uso del alcohol. De las respuestas de afrontamiento disponibles, las estrategias activas, focalizadas en el problema (Lazarus \& Folkman, 1984) son particularmente beneficiosas (Finney \& Moos, 1992). En contraste, los individuos que emplean la evitación, respuestas de afrontamiento focalizadas en la emoción, probablemente beberán en respuesta a las demandas (Billings y Moos, 1982; Finney \& Moos, 1992).

Marlatt (1988) ha relacionado el concepto de evaluación cognitiva y la teoría de la autoeficacia de Bandura, para entender las recaídas en personas con un consumo excesivo de alcohol. De acuerdo con la teoría de la autoeficacia (Bandura, 1997), cuando un paciente entra en una situación de alto riesgo por recaída alcohólica, se produce un proceso cognitivo mediante el cual evalúa su desempeño en dicha situación. El proceso de la evaluación cognitiva culmina con la formación de un juicio eficaz, el cual media en la conducta de consumo. La teoría de la autoeficacia sugiere que no es el consumo de alcohol per se el responsable de una recaída total.

En la evaluación cognitiva, el juicio de un individuo sobre el estrés relativo a las situaciones de alto riesgo es un tema importante a ser tratado. Marlatt (Annis \& Davis, 1985), utilizando el modelo de Lazarus (1966) sobre la evaluación primaria y secundaria en el pro- ceso de afrontamiento, ha relacionado la autoeficacia con el estrés percibido en una situación de alto riesgo. Observando la similitud entre los juicios de la autoeficacia y la evaluación secundaria, Marlatt argumenta que cuando el evento que está ocurriendo es significativo (evaluación primaria) y las respuestas de afrontamiento son consideradas como menos adecuadas para manejar la situación (evaluación secundaria), se experimenta el estrés psicológico. Bajo estas circunstancias, el individuo tiene altas probabilidades de recurrir a la bebida debido a que es la respuesta que dominó previamente en la jerarquía de afrontamiento. Por lo tanto, el proceso de evaluación tiene una doble función: primero, determinar cuál de las situaciones ha sido previamente manejada con un consumo excesivo de alcohol, y segundo, evaluar las expectativas actuales acerca de la adecuación de los recursos de afrontamiento.

Las estrategias de afrontamiento al estrés juegan un rol importante en aquellos sujetos que consumen regularmente alcohol y tabaco en varias formas: a) el uso es consistente o puede ser una parte integral de las respuestas de afrontamiento diseñadas para evitar, rechazar u olvidar el estresor; b) el uso tendrá mayor valor reforzante para personas que carecen de otros métodos para afrontar el estrés que para aquéllos que manejan un amplio rango de conductas de afrontamiento (Simons, Conger \& Whitbeck, 1988); y c) las es- 
trategias de afrontamiento, incluyendo el rechazo, evitación y distracción son métodos frecuentemente efectivos para tratar con las situaciones estresantes.

Los individuos ansiosos interpretan el significado de las situaciones, magnificando la proporción de las consecuencias posibles de temores propios percibidos. Los adolescentes se encuentran normalmente preocupados con los cambios de este período y la ansiedad adicional podría ser alentada por sobreestimaciones del costo de no tener éxito en las tareas de la adolescencia (Denoff, 1988).

Algunas investigaciones recientes se han centrado en las influencias positivas y negativas que podrían ejercer en la adaptación las distintas respuestas de afrontamiento. Los adolescentes, al igual que los adultos, manifiestan estrategias de afrontamiento tales como solución de problemas, regulación emocional y la evitación. Los hallazgos, consistentemente, han mostrado que las estrategias de solución de problemas están asociadas con el funcionamiento adaptativo y han sugerido que el afrontamiento centrado en la emoción, reflejo de una mala regulación emocional (preocupación, culparse a sí mismo, evidenciar de forma inapropiada las emociones negativas), se asocia con una mayor disfunción afectiva, conductual y social, y con las conductas de consumo de alcohol y de drogas (M. Windle \& R. Windle, 1996).

A partir de lo revisado nos propusimos llevar a cabo un estudio descripti- vo/correlacional acerca de las motivaciones y estilos de afrontamiento en jóvenes universitarios que consumen bebidas alcohólicas.

- Objetivo general.- Identificar las relaciones entre el consumo de bebidas alcohólicas, la tipología del bebedor, las motivaciones para hacerlo, los problemas relacionados con la bebida y los estilos de afrontamiento en un grupo de universitarios de uno y otro género que cursan los primeros años en una universidad privada en Lima.

- Objetivos específicos

- Describir las características del consumo de bebidas alcohólicas en función del tipo de bebida, cantidad, frecuencia, lugares de consumo y experiencias de exceso, así como presentar las características de la tipología del bebedor según género.

- Identificar los estilos de afrontamiento en los universitarios consumidores y no consumidores de bebidas alcohólicas.

- Identificar los motivos que subyacen al consumo de bebidas alcohólicas y los problemas relacionados con el consumo de éstas.

- Determinar las posibles relaciones entre la cantidad de alcohol consumido, los motivos para beber y los estilos de afrontamiento en los consumidores de bebidas alcohólicas según género. 
- Determinar las posibles relaciones entre la tipología del bebedor, los motivos para beber, los problemas relacionados y los estilos de afrontamiento según género.

En el presente estudio se consideran las siguientes definiciones conceptuales. Las definiciones operacionales se reflejan en los instrumentos respectivos.

\section{Definiciones conceptuales}

- Estilo de afrontamiento.- Se usa la conceptualización desarrollada por Lazarus y Folkman en 1986. Se define como una respuesta o conjunto de respuestas a eventos externos estresantes o depresivos, que la persona elabora en forma abierta o encubierta. El proceso se refiere a que la persona inicia sus esfuerzos pensando cómo puede enfrentar esta situación, para luego ejecutar la acción pertinente. Posteriormente, la persona también va a evaluar el afrontamiento ejecutado.

Carver et al. (1989) propusieron 13 escalas que se presentan a continuación:

- Escalas de afrontamiento orientado al problema

- Afrontamiento activo. Se define como el proceso de ejecutar acciones directas incrementando los esfuerzos con el fin de apartar o evitar el estresor o aminorar sus efectos negativos.
- La planificación. Implica pensar en cómo manejarse frente al estresor organizando estrategias de acción, pensando qué paso tomar y estableciendo cuál será la mejor forma de manejar el problema.

- La supresión de actividades competentes. Se refiere a dejar de lado o, incluso, dejar pasar otros proyectos o actividades evitando ser distraído, con la finalidad de poder manejar el estresor.

- La postergación del afrontamiento. Consiste en esperar hasta que se presente la oportunidad apropiada para actuar sobre el problema, evitando hacer algo prematuramente.

- La búsqueda de apoyo social por razones instrumentales. Consiste en buscar en los demás consejo, asistencia e información con el fin de solucionar el problema.

- Escalas de afrontamiento orientado a la emoción

- Búsqueda de apoyo social por razones emocionales. Consiste en buscar en los demás apoyo moral, empatía y comprensión con el fin de aliviar la reacción emocional negativa.

- La reinterpretación positiva y crecimiento. Consiste en evaluar el problema de manera positiva, otorgándole connotaciones favorables a la experiencia en sí misma y en otros casos asumiéndola 
como un aprendizaje para el futuro.

- La aceptación. Engloba dos aspectos durante el proceso de afrontamiento. El primero se realiza durante la fase de evaluación primaria cuando la persona acepta que la situación estresante realmente existe, y el segundo tiene lugar durante la evaluación secundaria y consiste en aceptar la realidad como un hecho con el que se tendrá que convivir ya que no se podrá modificar.

- La negación. Consiste en rehusarse a creer que el estresor existe o tratar de actuar pensando como si el estresor no fuera real.

- Acudir a la religión. Es la tendencia a volcarse a lo religioso en situaciones de estrés, con la intención de reducir la tensión existente.

- Otras escalas de afrontamiento

- Enfocar y liberar emociones. Consiste en focalizarse en las consecuencias emocionales desagradables que se experimenta expresando abiertamente estas emociones.

- Desentendimiento conductual. Consiste en la disminución del esfuerzo dirigido al estresor, dándose por vencido, sin intentar lograr las metas que el estresor está interfiriendo.

- Desentendimiento mental. Implica que la persona permite que una amplia variedad de actividades la distraigan de su evaluación de la dimensión conductual del problema o de la meta que el estresor está interfiriendo.

- Tipos de consumo de bebidas alcohólicas. Windle (1996) utiliza una tipología para evaluar las gradaciones con respecto a los niveles progresivos de involucramiento con el alcohol. El criterio utilizado para la categorización fue derivado de tres índices:

- El índice estándar de canti$\mathrm{dad} /$ frecuencia que refleja el número promedio de onzas de etanol consumidas por día.

- El número de episodios de beber en exceso: seis o más tragos en una ocasión.

- El número de problemas con el alcohol, principalmente consecuencias sociales adversas asociadas con su uso.

Los cortes para el índice cantidad/frecuencia y el número de días de beber excesivamente fueron consistentes con aquellos mencionados en la literatura (OMS, 1994a); uno de los cortes se basó en cinco o más problemas relacionados con el alcohol en los últimos seis meses, en aproximadamente el percentil 80 entre los sujetos que no fueron abstemios. Se presentan cinco categorías:

- Abstemio. No ha consumido un trago en los últimos 30 días. 
- Bebedor ligero. Ha consumido al menos un trago, pero menos de diez tragos en los últimos 30 días.

- Bebedor moderado. Ha consumido más que, o igual a diez tragos, pero menos de 45 tragos en los últimos 30 días.

- Bebedor intenso. Ha consumido 45 o más tragos en los últimos 30 días; o seis o más tragos en al menos cada una de las tres ocasiones en los últimos 30 días.

La Organización Mundial de la Salud (OMS, 1994a) define la conducta de beber excesivamente, en términos de excederse de cierto volumen diario (tres tragos al día), o cantidad por ocasión (cinco tragos en una ocasión, al menos una vez a la semana).

- Bebedor problema. Ha consumido 45 o más tragos en los últimos 30 días o ha consumido seis o más tragos al menos en cada una de las tres ocasiones en los últimos 30 días y ha reportado cinco o más problemas con el alcohol (faltar a la escuela debido al alcohol, desmayarse) en los últimos seis meses.

La Organización Mundial de la Salud define los problemas con el alcohol como cualquier rango de acompañantes adversos relacionados con el consumo de alcohol. Es importante señalar que "relacionado" no implica necesariamente causalidad. El uso del término puede referirse ya sea a un bebedor individual o a la sociedad, y fue acuñado por el Comité de Expertos de esta Organización en 1979.

- Motivos para beber. Son las razones dadas para el consumo de bebidas alcohólicas, que se pueden agrupar en motivos de estimulación, motivos de afrontamiento, motivos de conformidad y motivos sociales (Cooper, 1994).

Los motivos de estimulación son aquéllos generados internamente y por reforzamiento positivo. Se bebe para elevar el afecto positivo, el estado de ánimo o sentirse bien. Los motivos sociales son aquéllos generados externamente y por reforzamiento positivo. Se bebe en celebraciones sociales. Los motivos de afrontamiento son generados internamente y por reforzamiento negativo. Se bebe para reducir o regular las emociones negativas. Los motivos de conformidad son generados externamente y por reforzamiento negativo. Se bebe para evitar la censura social o rechazo.

\section{MÉTODO}

\section{Muestra}

El universo de estudio de la investigación estuvo conformado por alumnos de ambos géneros, con un rango de 
edades de 17 a 35 años. Ellos pertenecieron al primer año de Estudios Generales de una universidad privada en Lima.

La mitad de los participantes fueron mujeres y la otra mitad fueron hombres, con una media de 18,7 años y la mediana de 18. En cuanto al lugar de origen, la mayor parte de ellos nació en Lima $(86 \%)$ y vivía en ese momento con sus padres y hermanos (69\%). Asimismo, la mayoría ingresó a la universidad en el año 1997 (88\%) y se encontraba cursando el segundo ciclo de estudios generales $(77 \%)$. Por otro lado, casi una quinta parte de ellos se encontraba en tercer ciclo (19\%), llevando cursos de facultad.

El 68\% de la muestra reportó haber repetido cursos alguna vez, y de este grupo, $21 \%$ había repetido una vez, $20 \%$ dos veces y $11 \%$ tres veces. En relación con el rendimiento académico, el promedio ponderado se ubicó entre 11-12 para el $41 \%$ y $13-14$ para el $33 \%$.

En relación con el estrato social de procedencia y considerando las características sociodemográficas de la muestra, en la cual la mayoría de los sujetos procede de colegios particulares, vive en zonas residenciales, sus padres tienen educación superior y depende económicamente de éstos (Oficina de Admisión de la universidad, 1996), se podría presumir que ella es representativa de jóvenes que pertenecen a un estrato socioeconómico medio y medio alto.
Para fines de la investigación se utilizó un muestreo no probabilístico de tipo intencional. Se consideró los salones como unidades de muestreo y se seleccionó las aulas con mayor cantidad de estudiantes para garantizar la asistencia y lograr el tamaño estimado de la muestra.

Para la estimación del tamaño muestral se aplicó una fórmula de determinación del tamaño de muestra con uso del factor de corrección por población finita.

De acuerdo con la fórmula, la muestra tenía que ubicarse entre 396 y 400 casos para un margen de error de $2 \%$ y 3\%. Sin embargo, se logró reunir 440 casos válidos.

Es importante señalar que la muestra al ser no probabilística sólo puede considerarse como representativa de los estudiantes de los primeros años de educación superior de la universidad de donde fue extraída. Los resultados no pueden ser generalizados a los alumnos de primer año de otras universidades.

\section{Instrumentos}

Todos los instrumentos fueron sometidos al criterio de 12 jueces expertos en las áreas de estrés, afrontamiento y alcohol. Se solicitó la validez ítem/área y de adaptación linguíística de las pruebas al uso de la población hispanohablante universitaria de la ciudad capital. Posteriormente, se realizó un estudio piloto con 30 personas para verifi- 
car la comprensión de los reactivos de los instrumentos utilizados. Éstos fueron:

- Encuesta personal (EP). Se consignaron datos demográficos y académicos; los primeros incluyeron edad, sexo, lugar de nacimiento, tiempo de residencia en Lima y personas con las que vive; los segundos, el año de ingreso a la universidad, el ciclo de estudios, la carrera que pensaba estudiar, el promedio ponderado del ciclo anterior y cantidad (frecuencia) de cursos repetidos.

- Cuestionario de estilos de afrontamiento (COPE). Se utilizó el instrumento Cope (Coping Estimation) o estimación de afrontamiento, desarrollado por Carver, Scheier \& Weintraub (1989) en Estados Unidos. Fue construido basándose en dos modelos teóricos: el de Lazarus y el modelo de autorregulación conductual. Según los autores, esta escala pretende abarcar áreas de afrontamiento que no se consideraron en el Inventario de Tipos de Afrontamiento desarrollado por Folkman y Lazarus en 1980. Este instrumento fue adaptado por Casuso (1996) en población universitaria limeña.

Este instrumento está conformado por 52 ítems, con formato tipo Likert de cuatro puntos de calificación, los cuales se refieren a diferentes situaciones estresantes, dis- tribuidos en tres áreas. Se incorporaron conceptualmente 13 escalas o factores diferentes que correspondían a los 13 estilos de afrontamiento propuestos por Carver et al. en 1989.

Cada una de las escalas está conformada por cuatro ítems, los cuales se califican de acuerdo con una plantilla. Los puntajes obtenidos en cada ítem se suman con los correspondientes a cada subescala de manera que se obtienen 13 puntajes que permiten caracterizar un perfil de afrontamiento. Finalmente, se establece una jerarquía que permite determinar qué estilos de afrontamiento son los más utilizados.

A continuación se presentan los análisis psicométricos efectuados con los ítems de esta prueba y la consistencia interna en cada área de las escalas obtenidas en el presente estudio. Inicialmente, se realizó un análisis de correlación ítem/test el cual exigió una correlación mayor a 0.20 para que el ítem fuese aceptado (Kline, 1993). Posteriormente, se obtuvo la mediana de correlación ítem/test. Como índice de confiabilidad se utilizó el puntaje alfa de Cronbach, que se consideró significativo cuando era igual o mayor a 60 .

En cuanto al Cuestionario de Estilos de Afrontamiento (COPE), se realizó el análisis factorial por factores principales y con rotación oblimin y 
se encontró que de las 13 escalas sólo nueve se mantuvieron en su forma original y cuatro se reconstruyeron eliminándose un ítem. Sólo la escala 13 -Postergación del afrontamiento-, no se confirmó factorialmente debido a que dos ítems no se validaron; sin embargo, por congruencia teórica, se mantuvo como tal.

En el área Afrontamiento orientado al problema se validaron totalmente las siguientes escalas: planificación, búsqueda de apoyo social por razones instrumentales y supresión de actividades competentes, $\mathrm{y}$ todas presentaron una correlación ítem/ test y un puntaje alfa de Cronbach adecuados. La escala afrontamiento activo sólo se validó en tres de los cuatro ítems, pero se decidió mantenerla como tal. Las escalas que no se validaron totalmente fueron el afrontamiento activo y la postergación del afrontamiento, eliminándose un ítem de cada una. Sin embargo, para no afectar los puntajes totales de la matriz de correlación se multiplicó por $4 / 3$ cada escala.

En el área que corresponde al Afrontamiento orientado a la emoción se validaron las siguientes escalas: búsqueda de apoyo social por razones emocionales, acudir a la religión, reinterpretación positiva y crecimiento, y negación. Todos ellos presentan buena correlación ítem/test y alfa de Cronbach ade- cuadas. Sin embargo, en la escala de aceptación sólo se validaron tres ítems. Por ese motivo, también se tomó la decisión de multiplicar por $4 / 3$ esta escala.

En cuanto a la tercera área, correspondiente a Otros estilos de afrontamiento, se encontró que sólo una de las escalas se reproducía como en su forma original y ésta fue desentendimiento conductual. La escala desentendimiento mental se validó sólo con ítems, tomándose la misma decisión que en los casos anteriores.

- Encuesta sobre el consumo de bebidas alcohólicas para universitarios $(E C)$. En la presentación de los consumidores de bebidas alcohólicas se tomó en cuenta que la prevalencia de vida (usó alguna vez en su vida) no era un dato relevante para fines del estudio, dado que el alcohol es una droga lícita en nuestro medio. Por lo tanto, se consideró el reporte de consumo en los últimos seis meses y 30 días. Inicialmente se utilizó el período de los últimos seis meses para clasificar a los consumidores y no consumidores de bebidas alcohólicas. En los primeros se encontró que existían reportes de consumo exagerado en la cantidad de alcohol, creándose una categoría "consumo no válido". Para fines de la primera parte del reporte de resultados se hará referencia a los consumidores casos válidos y no válidos, exclu- 
yendo a los casos de reporte no válido para los análisis que incluyen la cantidad consumida. Se considera importante mencionar que para elaborar la tipología del bebedor de Windle (1996) se redefinió el criterio, considerándose como bebedores regulares a aquéllos que lo habían hecho en los últimos 30 días. Las preguntas estuvieron relacionadas con la cantidad y frecuencia con que se consumieron varios tipos de bebidas alcohólicas (cerveza, licor y vino) durante el último mes y en los últimos seis meses.

Una segunda medida del consumo fue la frecuencia de episodios de exceso, la cual se midió basándose en el número de veces durante el último mes y en los últimos seis meses en que la persona reportó haber consumido seis o más bebidas (Windle, 1996; M. Windle y R. Windle, 1996; OMS, 1994a). En la encuesta se incluyeron preguntas sobre el lugar de consumo de bebidas alcohólicas y el uso de otras sustancias. También se incluyeron preguntas acerca del consumo de bebidas alcohólicas por personas cercanas.

- Problemas relacionados con el alcohol (PA). Para medir los problemas asociados al consumo de bebidas alcohólicas que ocurrieron en los últimos seis meses, los participantes respondieron al cuestionario sobre problemas asociados al alco- hol de Windle (1996), traducido libremente para el presente estudio y con autorización del autor. Este cuestionario cuenta con 13 ítems que utilizan escalas Likert de cinco puntos (o rango de número de veces: $1-2,3-5,6-9,10$ a más). Los ítems fueron seleccionados sobre la base de su representación en estudios previos sobre consumo de bebidas alcohólicas en adolescentes (Barnes, 1990), y por su consistencia con el criterio diagnóstico de la tercera edición revisada del $M a$ nual diagnóstico y estadístico de desórdenes mentales (Asociación Psiquiátrica Americana, 1994).

Los ítems enunciados mencionan problemas -causados por la conducta de beber-, relacionados con los amigos, la familia, los profesores o las autoridades legales; faltar a clases, desmayarse o tener remordimientos al día siguiente de haber bebido. La confiabilidad medida a través del alfa de Cronbach fue 0.83 . Un análisis factorial de los ítems indicó un factor dominante (Windle, 1996).

En relación con el análisis psicométrico en el presente estudio del Cuestionario de Problemas (PA) asociados al consumo de bebidas alcohólicas de Windle, se formó una escala global con todos los ítems considerados por los autores (Windle, 1996). Se revisó la confiabilidad y se halló que ésta era adecuada (alfa = 
0.77). Al realizar el análisis factorial se obtuvieron cuatro factores. Debido a que la confiabilidad del cuarto factor fue 0.41 se tomó la decisión de eliminarlo y trabajar con tres factores, siendo éstos los siguientes:

El área uno con los ítems 3, 4, 5, 8, 9 (alfa de Cronbach $=0.63$ ), el área dos con los ítems 6, 9, 10, 12 (alfa de Cronbach $=0.68)$ y el área tres con los ítems 1 y 2 (alfa de Cronbach $=0.78$ ). Las tres áreas explican el $55,3 \%$ de la varianza. De acuerdo con el contenido de los ítems se dio nombre a cada una de las áreas. El área uno se denominó Problemas en las relaciones interpersonales; el área dos se llamó Problemas de conducta y el área tres se denominó Problemas con el área académica.

- Cuestionario para medir motivos para beber $(M B)$. Se utilizó el cuestionario motivos para beber (Drinking Motives Questionnaire) desarrollado por Cooper (1994), el que fue traducido libremente para fines del presente estudio. Éste cuenta con cuatro áreas: motivos de estimulación, motivos de afrontamiento, motivos de conformidad y motivos sociales. Cada área tiene cinco ítems que se evalúan en una escala que va del 1 al 6 (nunca, casi nunca, algunas veces, casi la mitad del tiempo, la mayor parte del tiempo y casi siempre). La consistencia in- terna estimada para esta escala fue de 0.75 (Windle, 1996).

En relación con la confiabilidad del cuestionario motivos para beber de Cooper (1994), en el presente estudio la confiabilidad de cada área fue adecuada. El área uno, motivos de estimulación, obtuvo un alfa de Cronbach de 0.89; el área dos, motivos de afrontamiento, un alfa de Cronbach de 0.87; el área tres, motivos de conformidad, un alfa de Cronbach de 0.90 y el área cuatro, motivos sociales, un alfa de Cronbach de 0.88 .

En la muestra estudiada los factores uno (Motivos de estimulación) y cuatro (Motivos sociales) tendieron a agruparse. Sin embargo, esto no impidió que se analicen los cuatro factores como lo postularon sus autores.

\section{Procedimiento}

La prueba piloto se aplicó en enero de 1998 a una muestra independiente de 30 estudiantes universitarios de ambos sexos de una universidad privada en Lima, mejorando la calidad de los instrumentos.

Para la obtención de la muestra se tomó contacto con las autoridades de la universidad y el coordinador de Estudios Generales, quien asignó las secciones a formar parte del estudio. Luego se tomó contacto con los/as profesores/as de las aulas asignadas para solicitar su colaboración. En todos los ca- 
sos se obtuvo una respuesta de interés hacia la investigación.

Las secciones asignadas fueron aquéllas que tenían mayor cantidad de alumnado con el fin de garantizar la asistencia y cubrir el tamaño de muestra requerido. Además, se eligieron cursos en los que no existía la posibilidad de que los alumnos estuvieran matriculados a la vez en más de uno, para así evitar la pérdida de casos por duplicación de información/datos.

La duración promedio de la encuesta fue de una hora. La secuencia de presentación de los instrumentos en el cuadernillo fue en el siguiente orden: a) encuesta personal, b) prueba Cope, c) encuesta sobre consumo de bebidas alcohólicas, problemas asociados y d) motivos para beber.

\section{Resultados}

Para el análisis de resultados se presentan, primero, los hallazgos descriptivos en relación con: a) la cantidad de alcohol consumido y la tipología del bebedor; b) los estilos de afrontamiento de consumidores y no consumidores y c) las motivaciones para beber y los problemas asociados con el consumo.
En segundo lugar, se analizan las correlaciones bivariadas encontradas entre la cantidad de alcohol consumido, la tipología del bebedor, motivos para beber, los problemas asociados y los estilos de afrontamiento.

Para facilitar la comprensión del reporte de la cantidad de licor (alcohol destilado) y vino en su equivalencia en botellas grandes de cerveza (véase la tabla $\mathrm{N}^{\mathrm{o}} 1$ ).

\section{Resultados descriptivos del consumo en los últimos seis meses}

Se puede observar que tanto hombres como mujeres consumieron bebidas alcohólicas, siendo más del doble el número de mujeres que no consumió. Casi la totalidad de los estudiantes que consumió bebidas alcohólicas lo hizo acompañado (99\%), generalmente de sus amigos $(95 \%)$ y en fiestas $(92 \%)$; con menor frecuencia en pubs/discotecas $(75 \%)$ o en casa de los amigos $(65 \%)$. La mayoría de los universitarios consumió cerveza (94\%) y licor $(90 \%)$; y un porcentaje menor de ellos bebió vino (72\%). En general no se consumió una sola bebida alcohólica, los que consumieron cerveza también

Tabla № 1

Equivalencia de la bebidas alcohólicas

\begin{tabular}{lcc}
\hline Contenido de alcohol puro & $150 \mathrm{ml}$ de etanol & $300 \mathrm{ml}$ de etanol \\
\hline Cerveza $\left(5^{\circ} \mathrm{Gl}\right)$ & $41 / 2$ botellas & 9 botellas \\
Vino $\left(10-12^{\circ} \mathrm{Gl}\right)$ & 2 a $21 / 2$ botellas & $4-5$ botellas \\
Destilados (ron, whisky, pisco, cañazo) $\left(45^{\circ}-50 \mathrm{Gl}\right)$ & $1 / 2$ botella & 1 botella \\
\hline
\end{tabular}

Nota: Tomado del MALT-F (test de alcoholismo de Munich, Alemania). 
consumieron licor y vino. Asimismo, el mayor consumo tendió a ser de cerveza con licor o de licor con vino. Esto quiere decir que cuando se consume cerveza no se tiende a consumir vino. Respecto de la cantidad consumida, en el caso de los hombres, se encontró que quienes consumieron más cerveza, también consumieron más licor y vino. En el caso de las mujeres, se repitió el patrón, a excepción de la cantidad de vino que fue asociada con el licor. Es decir, las mujeres que consumieron más cerveza consumieron también más licor, pero no vino; mientras que aquéllas que consumieron más licor también consumieron vino.

Se encontró que ambos géneros consumieron todas las bebidas alcohólicas, siendo menor el caso de la cerveza en las mujeres $(88 \%)$. Sin embargo, fue ligeramente mayor para éstas en el caso del licor (91\%) y descendió en el caso del vino (69\%), (véase tabla $\mathrm{N}^{\circ} 2$ ).
Se encontró que no se puede trabajar con los promedios de consumo de cerveza, licor y vino debido a que dichos promedios disfrazan importantes diferencias en la frecuencia de consumo de cada una de estas bebidas. Por lo tanto, se prefirió hacer un análisis separado por cada bebida alcohólica, (véase tabla $\mathrm{N}^{\circ}$ 2).

En cuanto a la frecuencia de consumo de la cerveza se encontró que lo más usual era consumir entre una y cuatro ocasiones por mes (53\%), (véase tabla $\mathrm{N}^{\mathrm{o}} 3$ ), especialmente una vez por semana (26\%). Se observó que es poco usual consumir más de una vez por semana (11\%) y que casi ningún joven consumió más de tres días a la semana (2\%).

La unidad de bebida que consumieron los hombres fue en botellas grandes $(620 \mathrm{ml})$ o chicas $(355 \mathrm{ml})$, en vez de vasos o botellas de $1.100 \mathrm{ml}$; mientras que las mujeres consumieron en botellas chicas o vasos. Sin embargo, más im-

Tabla № 2

Consumo de bebidas alcohólicas según tipo de bebida por género en porcentajes

\begin{tabular}{lcccccccccc}
\hline & & \multicolumn{1}{c}{ Cerveza } & \multicolumn{4}{c}{ Tipo de bebida alcohólica } \\
Licor* \\
& H & $\mathbf{M}$ & $\mathbf{T}$ & $\mathbf{H}$ & $\mathbf{M}$ & $\mathbf{T}$ & $\mathbf{H}$ & $\begin{array}{c}\text { Vino } \\
\mathbf{M}\end{array}$ & $\mathbf{T}$ \\
\hline & & & & & & & & & \\
Consumo & $(209)$ & $(203)$ & $(412)^{\mathrm{a}}$ & $(212)$ & $(203)$ & $(415)^{\mathrm{b}}$ & $(204)$ & $(201)$ & $(405)^{\mathrm{c}}$ \\
Sí & & & & & & & & & \\
No & 99 & 88 & 94 & 89 & 91 & 90 & 75 & 69 & 72 \\
\hline
\end{tabular}

Nota: $\mathrm{N}=417 .{ }^{*}$ Ron, vodka, whisky y cocteles; a hubo cinco casos incompletos; ${ }^{b}$ hubo dos casos incompletos; c hubo 12 casos incompletos. 
Tabla № 3

Frecuencia del consumo por tipo de bebida alcohólica en porcentajes (\%)

\begin{tabular}{|c|c|c|c|c|c|c|c|}
\hline \multirow[b]{3}{*}{ Frecuencia } & \multicolumn{6}{|c|}{ Tipo de bebida alcohólica } & \multirow{3}{*}{$\begin{array}{c}\text { Promedio }^{\mathrm{d}} \\
\%\end{array}$} \\
\hline & \multicolumn{2}{|c|}{$\begin{array}{c}\text { Cerveza } \\
(412)^{\mathrm{a}}\end{array}$} & \multicolumn{2}{|c|}{$\begin{array}{l}\text { Licor } \\
(415)^{b}\end{array}$} & \multicolumn{2}{|c|}{$\begin{array}{c}\text { Vino } \\
(405)^{c}\end{array}$} & \\
\hline & $\%$ & $\%$ Ac. & $\%$ & $\%$ Ac. & $\%$ & $\%$ Ac. & \\
\hline Nunca & 6,3 & 6,3 & 10,1 & 10,1 & 28,4 & 28,4 & 14 \\
\hline Algunas veces & 27,7 & 34 & 30,4 & 40,5 & 40,7 & 69,1 & 33 \\
\hline Una vez al mes & 9,4 & 43,4 & 14,2 & 54,7 & 13,1 & 82,2 & 12 \\
\hline 2-3 d. al mes & 17,5 & 61,2 & 22,2 & 76,9 & 8,9 & 91,1 & 16 \\
\hline Una vez por semana & 26,2 & 87,4 & 18,3 & 95,2 & 5,9 & 97 & 17 \\
\hline 2-3 d. por semana & 10,9 & 98,3 & 4,1 & 99,3 & 1,7 & 98,8 & 6 \\
\hline 4-5 d. por semana & 1,7 & 100 & 0,7 & 100 & 0,5 & 99,3 & 1 \\
\hline Todos los días & - & - & - & - & 0,7 & 100 & 0,5 \\
\hline
\end{tabular}

Nota: a 5 casos incompletos; ${ }^{b} 2$ casos incompletos, ${ }^{c} 12$ casos incompletos; d Promedio de los porcentajes;

$\%$ Ac. = porcentaje acumulado.

portante que la unidad de bebida fue la cantidad ingerida, por lo que mereció un análisis aparte. El consumo típico en una ocasión promedio en botellas grandes $(620 \mathrm{ml})$ fue de dos botellas tanto para hombres como para las mujeres.

Un $60 \%$ reportó haber tenido de una a dos experiencias de excesos, es decir haber consumido seis o más bebidas de cerveza en una ocasión en los últimos seis meses. Mientras un tercio de los consumidores de cerveza reportó no haberlas tenido y un $7 \%$, más de tres excesos.

Para uniformizar el reporte de la cantidad consumida del licor y vino, se presenta su equivalencia en mililitros de cerveza y botellas grandes de $620 \mathrm{ml}$.

En cuanto al consumo de licor (ron, vodka, whisky y cocteles) se encontró que lo más usual era consumir entre una y cuatro ocasiones por mes (55\%), especialmente de dos a tres días al mes (22\%). Se observó que era poco usual consumir más de una vez por semana (4\%) y casi ningún joven reportó haber consumido más de tres días a la semana $(0,7 \%)$. Se hallaron diferencias por género; mientras que el $62 \%$ de los hombres reportó consumir entre una y más ocasiones al mes, el $47 \%$ de mujeres reportó esta frecuencia. El consumo típico en una ocasión promedio equivalente en botellas grandes de cerveza, fue de tres botellas. Esto se mantuvo para los hombres, mientras que las mujeres reportaron consumir el equivalente a dos botellas.

A diferencia del consumo de cerveza, un $40 \%$ de los consumidores de licor reportó no haber tenido experiencias de exceso con esta bebida; mientras que la mayoría (57\%) manifestó haber tenido entre una a dos de esas experiencias, y 
sólo un tres por ciento reportó tres o más de ellas.

En relación con el consumo de vino $41 \%$ reportó tomarlo algunas veces. Un $28 \%$ consumió vino entre una y cuatro ocasiones por mes, predominando el grupo que bebió vino una vez al mes (13\%). Se observó que es poco usual consumir más de una vez por semana (2\%) y casi ningún joven consumió más de tres días a la semana $(0,5 \%)$. Sólo en este tipo de bebida se reportó el consumo todos los días $(0,5 \%)$ (véase tabla $\mathrm{N}^{\mathrm{o}} 3$ ). El consumo promedio de vino equivalente en botellas grandes de cerveza fue una botella y esto se mantuvo tanto para hombres como para mujeres. La mayoría reportó no haber tenido experiencias de excesos con el vino $(56 \%$ los hombres y $67 \%$ las mujeres).
Cabe señalar que la frecuencia de consumo del vino siguió un patrón bastante distinto al consumo de cerveza y licor.

Sobre la cantidad de cerveza consumida por mes, en promedio, los hombres consumieron casi el doble que las mujeres: un promedio de $9.300 \mathrm{ml} \mathrm{de}$ cerveza (equivalente a 15 botellas de $620 \mathrm{ml}$ ) (véase tabla $\mathrm{N}^{\circ} 4$ ). Desde un análisis de percentiles, un cuarto de los hombres bebieron hasta dos cervezas y las mujeres un tercio de botella. La mitad de los hombres llegó a tomar hasta ocho botellas, es decir, ocho veces más que las mujeres. A esto se suma un $25 \%$ de hombres que llegó a tomar hasta 20 botellas. Mientras que las mujeres no llegaron a consumir más de siete (véase tabla $\mathrm{N}^{\mathrm{o}} 5$ ).

Respecto del licor, en promedio, los hombres consumieron más que las mu-

Tabla № 4

Promedio de consumo mensual de bebida alcohólica según género

\begin{tabular}{|c|c|c|c|c|}
\hline Bebida & & $\begin{array}{c}\mathrm{H} \\
(193)^{\mathrm{a}}\end{array}$ & $\begin{array}{c}\text { Género } \\
\mathrm{M} \\
(194)^{\mathrm{b}}\end{array}$ & $\begin{array}{c}\mathrm{T} \\
(398)^{\mathrm{C}}\end{array}$ \\
\hline \multirow[t]{3}{*}{ Cerveza } & $M$ & 15 & 6 & 11 \\
\hline & $\mathrm{Md}$ & 8 & 1 & 6 \\
\hline & DS & 19 & 12 & 13 \\
\hline \multirow[t]{3}{*}{ Licor } & $M$ & 9 & 6 & 9 \\
\hline & $\mathrm{Md}$ & 5 & 2 & 5 \\
\hline & DS & 14 & 10 & 13 \\
\hline \multirow[t]{3}{*}{ Vino } & $M$ & 3 & 2 & 3 \\
\hline & Md & 0,6 & 0,3 & 0,9 \\
\hline & DS & 6 & 3,6 & 6 \\
\hline
\end{tabular}

Nota: El consumo se ha homogeneizado a la unidad equivalente en número de botellas de $620 \mathrm{ml}$. consumidas al mes. a 6 casos incompletos; b 5 casos incompletos; ${ }^{c}$ Total de casos con reporte de consumo válido en todos los siguientes cuadros. 
jeres: un promedio de $5.580 \mathrm{ml}$ de cerveza (equivalente a nueve botellas de 620ml) (véase tabla $\mathrm{N}^{\circ} 4$ ). Desde un análisis de los percentiles, un tercio de las mujeres y un cuarto de los hombres bebieron hasta el equivalente a una botella de cerveza. La mitad de los hombres llegó a tomar hasta el equivalente de cinco botellas grandes, es decir, el doble que las mujeres. A esto se adiciona un $25 \%$ de hombres que llegó a tomar hasta el equivalente a ocho botellas de cerveza (véase tabla $\mathrm{N}^{\circ}$ 5).

En relación con el vino, la cantidad consumida fue muy similar para hombres y mujeres; en promedio, los hombres consumieron ligeramente más que las mujeres: $1.860 \mathrm{ml}$ de cerveza (equivalente a tres botellas de $620 \mathrm{ml}$. de cerveza). Desde un análisis de los percentiles, un cuarto de hombres y mujeres no ha consumido vino. La mitad de hombres y mujeres no llegó a consumir el equivalente a una botella de $620 \mathrm{ml}$ de cerveza. Sin embargo, un $25 \%$ de varones consumió el doble que las mujeres (véase tabla $\mathrm{N}^{\circ} 5$ ).
Entre aquéllos que consumían bebidas alcohólicas, poco más de la mitad consumía tabaco (58\%) y en este caso el $42 \%$ lo hacía siempre y el $25 \%$ muchas veces. Un $13 \%$ reportó consumo de marihuana y, entre ellos, la mayoría fueron hombres $(17 \%)$. Un pequeño grupo manifestó consumir cocaína (5\%), siendo el grupo de los hombres el de mayor consumo reportado (8\%).

\section{Análisis descriptivo de la tipología del bebedor}

En relación con la tipología del bebedor establecida por Windle (1996), se hicieron los cálculos necesarios y se halló que la mitad de los bebedores se ubicaban entre ligeros y moderados (55\%), seguidos por los bebedores problemáticos (21\%), los abstemios en 30 días $(14 \%)$ y por último los bebedores intensos (9\%). Se observó que esta tipología presentaba algunas diferencias por género, siendo mayor el porcentaje de mujeres abstemias en 30 días (20\%) que el de los hombres (8\%), y menor el porcentaje de bebedoras problemáticas (13\%) que el de bebedores problemáti-

Tabla № 5

Cantidad de bebida alcohólica consumida por mes en percentiles, según género

\begin{tabular}{lcccccccccccc}
\hline & \multicolumn{1}{c}{} & \multicolumn{1}{c}{ Cerveza } & \multicolumn{4}{c}{$\begin{array}{c}\text { Tipo de bebida alcohólica } \\
\text { Licor }\end{array}$} \\
\hline & P25 & P50 & P75 & Min. & P25 & P50 & P75 & Mín. & P25 & P50 & P75 Mín. \\
$\mathrm{H}^{\mathrm{a}}$ & 2 & 8 & 20 & 0 & 1 & 5 & 12 & 0 & 0 & 0.6 & 2 & 0 \\
$\mathrm{M}^{\mathrm{b}}$ & 0.3 & 1 & 7 & 0 & 0.3 & 2 & 8 & 0 & 0 & 0.3 & 1 & 0 \\
\hline
\end{tabular}

Nota. ${ }^{a} n=193,6$ casos incompletos; ${ }^{b} n=194,5$ casos incompletos. 
$\cos (31 \%)$. Se presentaron más bebedoras ligeras (31\%) y se igualó el consumo en la categoría bebedor moderado e intenso (véase tabla $\mathrm{N}^{\circ} 6$ ).

De acuerdo con la tipología del bebedor, en todos los casos, las bebidas alcohólicas eran consumidas en compañía de otras personas. A medida que el consumo de bebidas alcohólicas pasaba de moderado a intenso y problemático, se consumía con mayor frecuencia tabaco $(65 \%, 75 \%$ y $74 \%$, respectivamente). Se observó que el consumo de marihuana fue más frecuente entre los bebedores problemáticos $(25 \%)$ e intensos (18\%). El consumo de cocaína se presentó entre los bebedores moderados, intensos y problemáticos $(7 \%$, $7 \%$ y $8 \%$, respectivamente).

Los bebedores intensos fueron quienes más reportaron el consumo de bebidas alcohólicas en su entorno (92\%), seguidos por los problemáticos $(88 \%)$ y los moderados (87\%). Entre los abstemios y ligeros la frecuencia de consumo disminuyó (75\% y $70 \%$, respectivamente).

\section{Estilos de afrontamiento en los consumidores y no consumidores de bebidas alcohólicas}

Usando la prueba $\mathrm{T}$ de Student para diferencias entre muestras independientes, es decir entre consumidores y no consumidores en los últimos seis meses, se halló que las diferencias en el uso de los estilos de afrontamiento se daban para los orientados a la emoción, y los no consumidores lo utilizaron más. Sin embargo, con los estilos orientados al problema y otros estilos de afrontamiento no se presentaron diferencias entre consumidores y no consumidores de bebidas alcohólicas. En cuanto a las escalas de afrontamiento, sólo se presentaron diferencias en el uso de acudir a la religión y reinterpretación positiva, siendo los no consumidores quienes lo utilizaron más.

En lo que respecta a los estilos de afrontamiento, los porcentajes generales permitieron comparar los estilos de afrontamiento orientados al problema, a la emoción, y otros estilos. Las per-

Tabla № 6

Distribución de los tipos de bebedores

\begin{tabular}{lrrrrrc}
\hline $\begin{array}{l}\text { Tipo de } \\
\text { bebedor }\end{array}$ & \multicolumn{2}{c}{$\mathbf{H}$} & \multicolumn{2}{c}{$\mathbf{M}$} & \multicolumn{2}{c}{$\mathbf{T}$} \\
$\mathbf{( 2 0 6}^{\mathbf{a}}$ & $f$ & $\%$ & $f$ & $\%$ & \multicolumn{2}{c}{$\mathbf{( 4 2 1 )}^{\mathbf{c}}$} \\
\hline & 17 & 8,3 & 42 & 19,5 & 59 & $\%$ \\
Abstemio 30d. & 43 & 20,9 & 67 & 31,2 & 110 & 26,1 \\
Ligero & 62 & 30,1 & 61 & 28,4 & 123 & 29,2 \\
Moderado & 20 & 9,7 & 18 & 8,4 & 38 & 9 \\
Intenso & 64 & 31,1 & 27 & 12,6 & 91 & 21,6 \\
Problemático & & &
\end{tabular}

Nota. ${ }^{a} 13$ casos incompletos; ${ }^{b} 6$ casos incompletos; ${ }^{c} 19$ casos incompletos. 
sonas que consumían bebidas alcohólicas refirieron que utilizaban preferentemente estilos de afrontamiento orientados a la emoción (21\%), seguidos por los que estaban orientados al problema (15\%) y, por último, otros estilos $(5 \%)$.

Se observó algunas diferencias de acuerdo con el género. El estilo de afrontamiento más utilizado se presentó en el caso de las mujeres que consumían bebidas alcohólicas y fue el orientado a la emoción (28\%). Tanto los hombres como las mujeres refirieron utilizar estilos orientados al problema (14,4\% y $14,9 \%$, respectivamente). Finalmente, se reportó el uso de otros estilos de afrontamiento y esto fue algo mayor en el caso de las mujeres que en los hombres $(6,7 \%$ y $3,8 \%$, respectivamente).

Los estudiantes que no consumían bebidas alcohólicas refirieron no utilizar preferentemente ningún estilo de afrontamiento. Usando criterios descriptivos, se usaron los dos estilos orientados al problema y a la emoción.

\section{Motivaciones para beber y problemas asociados al consumo de bebidas alcohólicas}

Los motivos más importantes para beber fueron sociales $(2,98)$ y de estimulación $(2,56)$. Sin embargo, no se reportó ningún motivo especialmente destacable. Esto se presentó tanto en hombres como en mujeres (véase tabla $\mathrm{N}^{\mathrm{o}} 7$ ).
Tabla № 7 Motivos para beber

\begin{tabular}{lcc}
\hline Motivos para beber & $\mathbf{M}^{\mathbf{a}}$ & DE \\
\hline Motivos sociales & 2.98 & 1.10 \\
Motivos de estimulación & 2.56 & 1.14 \\
Motivos de afrontamiento & 1.82 & 0.82 \\
Motivos de conformidad & 1.50 & 0.61 \\
\hline
\end{tabular}

Nota: $n=371.27$ casos no completaron este cuestionario. a rango creciente de frecuencia de 1 a 6 , donde $1=$ nunca y $6=$ siempre.

En cuanto a los problemas asociados al consumo de bebidas alcohólicas se encontró que los más importantes fueron los de relaciones interpersonales $(1,5)$. Asimismo, casi nunca se presentaron problemas de conducta o similares en el área académica (véase tabla No 8 ).

\section{Tabla № 8}

Problemas asociados al consumo de bebidas alcohólicas

\begin{tabular}{lcc}
\hline Tipo de problema & M & DE \\
\hline $\begin{array}{l}\text { Relaciones } \\
\text { interpersonales }\end{array}$ & 1.5 & 0.47 \\
Problemas en el área & & \\
académica & 1.4 & 0.67 \\
Problemas de conducta & 1.2 & 0.36 \\
\hline
\end{tabular}

Nota. $\mathrm{n}=391.7$ casos no completaron el cuestionario. Rango de 1-5, donde $1=$ nunca y 5 = frecuentemente.

\section{Correlaciones entre la cantidad de} alcohol consumida, los motivos para beber, los problemas relacionados $y$ los estilos de afrontamiento

Se realizó un análisis de correlación entre la cantidad de alcohol consumida, los motivos para beber, los problemas relacionados y los estilos de afronta- 
miento en los consumidores de bebidas alcohólicas. Para identificar las correlaciones que luego serían objeto de un análisis más detallado, se aplicó primero un criterio de significación estadística, a un nivel $p<.05$. Posteriormente, se aplicó el criterio de clasificación de Cohen para garantizar correlaciones de magnitudes relevantes $(r>$.2) (Cohen, 1988). Se considera importante señalar que no se ha aplicado este criterio en todos los casos, debido a que en algunos de ellos existían razones teóricas que nos permitían presumir relaciones múltiples. Es decir, no necesariamente grandes en magnitud, pero sí en cantidad.

Se presentaron relaciones entre los motivos de estimulación, afrontamiento y sociales con la cantidad de alcohol consumido en los últimos seis meses. Estas asociaciones también se mantuvieron para el reporte de alcohol consumido en los últimos 30 días. Los motivos de conformidad no presentaron re- laciones con el total de alcohol consumido en seis meses ni en los últimos 30 días (véase tabla $\mathrm{N}^{\circ}$ 9). Sin embargo, el análisis de regresión y la correlación parcial que se presenta más adelante, mostró que los motivos de conformidad sí tienen correlación significativa con la cantidad de alcohol consumido cuando se consideran simultáneamente con los otros motivos.

Las mujeres que bebían por motivos de estimulación, afrontamiento y sociales tendieron a beber más que los hombres en los últimos seis meses. En el caso del consumo en 30 días, se observó que las mujeres que bebían por motivos de afrontamiento, bebían más que los hombres. Sin embargo, se observó que entre quienes bebían por motivos sociales, los hombres reportaron beber más que las mujeres. Asimismo, entre aquéllos que bebían por motivos de estimulación, los hombres bebieron ligeramente más que las mujeres.

Tabla № 9

Correlaciones entre los motivos para beber y total de alcohol consumido

\section{Motivaciones para beber}

\begin{tabular}{|c|c|c|c|c|c|c|c|c|c|c|c|c|}
\hline & \multicolumn{3}{|c|}{$\begin{array}{l}\text { Motivos de } \\
\text { estimulación }\end{array}$} & \multicolumn{3}{|c|}{$\begin{array}{l}\text { Motivos de } \\
\text { afrontamiento }\end{array}$} & \multicolumn{3}{|c|}{$\begin{array}{l}\text { Motivos de } \\
\text { conformidad }\end{array}$} & \multicolumn{3}{|c|}{$\begin{array}{l}\text { Motivos } \\
\text { sociales }\end{array}$} \\
\hline & $\mathbf{H}$ & $\mathbf{M}$ & $T$ & $\mathbf{H}$ & $\mathbf{M}$ & $\mathbf{T}$ & $\mathbf{H}$ & $\mathbf{M}$ & $\mathbf{T}$ & $\mathbf{H}$ & $\mathbf{M}$ & $\mathbf{T}$ \\
\hline $\begin{array}{l}\text { Total alcohol en } \\
\text { seis meses } \\
\text { Total de alcohol en }\end{array}$ & $34^{*}$ & $46^{*}$ & $40^{*}$ & $17^{*}$ & $45^{*}$ & $24^{*}$ & -.02 & .03 & .00 & $.29^{*}$ & $.35^{\star}$ & $.35^{*}$ \\
\hline 30 días & $.43^{*}$ & $.40^{\star}$ & $.43^{*}$ & $.26^{*}$ & $.38^{*}$ & $.25^{*}$ & -.02 & -.08 & -.04 & $.38^{*}$ & $.30^{*}$ & $.39^{*}$ \\
\hline
\end{tabular}

Nota. ${ }^{*} \mathrm{p}<.05 . \mathrm{H}=$ hombre. $\mathrm{M}=$ mujer. $\mathrm{T}=$ correlación global. 
Correlaciones entre los motivos para beber y el total de alcohol consumido

Se halló que quienes utilizaban otros estilos de afrontamiento -tanto hombres y mujeres- tendían a consumir más bebidas alcohólicas en los últimos 30 días. Esta relación se mantuvo en el caso de las mujeres en los últimos seis meses. Entre estos estilos, la escala enfocar y liberar emociones presentó mayor relación tanto en 30 días como en seis meses. Por otra parte, las mujeres que utilizaban el desentendimiento mental reportaron más consumo de alcohol en los últimos seis meses (véase tabla $\mathrm{N}^{\circ} 10$ ).

No se halló relación con los estilos orientados al problema. A nivel de escalas se observó que los hombres que utilizaban la búsqueda de apoyo social por razones instrumentales, tendían a consumir más alcohol. Tampoco se encontró relación con el estilo orientado a la emoción. Sin embargo, a nivel de escalas se observó que los hombres que utilizaron la aceptación habían bebido más en los últimos seis meses.

Por otro lado, se reportó una relación inversa con acudir a la religión. No obstante, esta relación desapareció en los últimos seis meses, tanto para los hombres como para las mujeres; manteniéndose, en el caso de las mujeres, en el consumo de alcohol en 30 días.

Se halló una relación entre la cantidad de alcohol consumido en 30 días y en los últimos seis meses y los problemas asociados al consumo, tanto en hombres como en mujeres; siendo esta relación más fuerte para los hombres en los últimos 30 días.

\section{Correlaciones entre la tipología del bebedor, los motivos para beber, los problemas relacionados y los estilos de afrontamiento}

La tipología del bebedor que permite clasificar a los bebedores en un rango que va desde abstemios en los últimos

Tabla № 10

Correlaciones entre los estilos de afrontamiento y la cantidad de alcohol

\begin{tabular}{|c|c|c|c|c|c|c|}
\hline \multirow[b]{3}{*}{ Estilos } & \multicolumn{6}{|c|}{ Total de alcohol } \\
\hline & \multicolumn{3}{|c|}{30 días } & \multicolumn{3}{|c|}{6 meses } \\
\hline & $\mathbf{H}$ & $\mathbf{M}$ & $\mathbf{T}$ & $\mathbf{H}$ & $\mathbf{M}$ & $\mathbf{T}$ \\
\hline Estilos orientados al problema & .05 & -.03 & .01 & .12 & .04 & .08 \\
\hline A. social-instrumental & $.23^{*}$ & -.06 & .10 & $.22^{*}$ & -.01 & -.07 \\
\hline Orientado a la emoción & .07 & -.01 & -.03 & .12 & -.01 & .00 \\
\hline Aceptación & .12 & .06 & .08 & $.19^{*}$ & -.09 & $.11^{*}$ \\
\hline A. religión & .11 & $-.16^{*}$ & $-.19^{\star}$ & -.03 & .09 & $-.12^{*}$ \\
\hline Otros estilos & $.17^{*}$ & $.16^{*}$ & .08 & .14 & $.17^{*}$ & .08 \\
\hline Enfocar y liberar emociones & $.22^{*}$ & $.24^{\star}$ & $.12^{*}$ & $.26^{*}$ & $.18^{*}$ & $.14^{*}$ \\
\hline Desentendimiento mental & .08 & .06 & .03 & .05 & $.15^{\star}$ & .06 \\
\hline
\end{tabular}

Nota. ${ }^{*} p>$.05. $\mathrm{H}=$ hombre, $\mathrm{M}=$ mujer. $\mathrm{T}$ = correlación global. 
30 días hasta los bebedores problemáticos, presentó una fuerte relación con los problemas asociados al consumo y las motivaciones para beber como los motivos de estimulación, los motivos sociales, los motivos de afrontamiento, a excepción de los motivos de conformidad en donde la correlación no fue significativa. Al introducir la variable género se observó que esta tendencia se mantenía (véase tabla $\mathrm{N}^{\circ} 11$ ). Sin embargo, se presentaron algunas diferencias en las mujeres. En la medida en que el consumo se hacía más problemático éste se hacía como una forma de estimularse, de afrontar sus dificultades y por motivos sociales. Mientras que los hombres cuyo consumo se hacía más problemático lo hacían más por razones de estimulación, sociales y de afrontamiento. En relación con los problemas reportados, se obtuvo que los hombres tendían a presentar más problemas de conducta en la medida en que su consumo se hacía más problemático, así como también problemas de relaciones interpersonales o académicos. Las mujeres presentaban más problemas interpersonales, académicos y de conducta. Estas correlaciones se resumen en el siguiente cuadro.
Tabla № 11

Correlaciones entre los motivos para beber, los problemas asociados y la tipología del bebedor, según género

\begin{tabular}{|c|c|c|c|}
\hline \multirow[b]{2}{*}{ Variables } & \multicolumn{3}{|c|}{ Tipología del bebedor } \\
\hline & $\mathbf{H}$ & $\mathbf{M}$ & $\mathbf{T}$ \\
\hline \multicolumn{4}{|l|}{ Motivos } \\
\hline Estimulación & $.46^{*}$ & $.57^{*}$ & .53 \\
\hline Afrontamiento & $.29^{*}$ & $.51^{*}$ & -.37 \\
\hline Sociales & $.38^{*}$ & $.51^{*}$ & $.47^{*}$ \\
\hline \multicolumn{4}{|l|}{ Problemas } \\
\hline Problema & $.58^{*}$ & $.57^{\star}$ & $.59^{*}$ \\
\hline Interpersonales & $.42^{*}$ & $.45^{*}$ & $.45^{*}$ \\
\hline Conducta & $.48^{*}$ & $.32^{*}$ & $.45^{*}$ \\
\hline Académicos & $.41^{*}$ & $.39^{*}$ & $.40^{*}$ \\
\hline
\end{tabular}

Nota. ${ }^{*} \mathrm{p}>.05 . \mathrm{H}=$ hombre, $\mathrm{M}=$ mujer. $\mathrm{T}=$ correlación global.

La tipología del bebedor no se relacionó con ningún estilo de afrontamiento en particular. Sin embargo, se hallaron algunas diferencias según género. Las mujeres que más bebían o que tenían un consumo más problemático, tendían a utilizar más otros estilos de afrontamiento, tales como enfocar y liberar emociones y el desentendimiento mental.

A nivel de escalas de afrontamiento, los universitarios cuyo consumo se hacía más problemático, tendían menos a acudir a la religión como una forma de afrontamiento. Esto se mantuvo tanto para hombres como para las mujeres. Asimismo, en la medida en que el consumo se hacía más problemático los universitarios tendían a enfocar y liberar emociones. Los hombres que tendían a consumir más, utilizaban menos 
la postergación del afrontamiento. Se considera importante mencionar que las demás escalas de afrontamiento no correlacionaron significativamente $(p<.05)$ (véase tabla $\left.\mathrm{N}^{\circ} 12\right)$.

Tabla № 12

Correlaciones entre la tipología del bebedor y los estilos de afrontamiento según género

\begin{tabular}{lccc}
\hline & \multicolumn{3}{c}{ Tipología del bebedor } \\
Variables & $\mathbf{H}$ & $\mathbf{M}$ & $\mathbf{T}$ \\
\hline Enfocado en la & & & \\
emoción & -.09 & -.02 & -.07 \\
$\begin{array}{l}\text { Religión } \\
\text { Enfocado en el }\end{array}$ & $-.21^{*}$ & $-.24^{\star}$ & $-.22^{*}$ \\
problema & -.07 & .02 & .00 \\
$\begin{array}{l}\text { Postergación } \\
\text { Otros estilos }\end{array}$ & $-.16^{\star}$ & -.08 & -.08 \\
$\begin{array}{l}\text { Enfocar y liberar } \\
\text { emociones }\end{array}$ & .09 & $.22^{*}$ & .01 \\
$\begin{array}{l}\text { Desentendimiento } \\
\text { mental }\end{array}$ & .14 & $.23^{*}$ & $.14^{*}$ \\
\hline
\end{tabular}

Nota. ${ }^{*} p<.05$.

A modo de resumen se realizó la correlación parcial controlada por género y la clasificación de la tipología del bebedor de Windle, y se halló que los ca- sos en donde hubo al menos un $10 \%$ de varianza que podría atribuirse al agregado de estas dos variables, fueron los motivos de estimulación y sociales con los problemas en general, problemas interpersonales y de conducta. En la tabla $\mathrm{N}^{\circ} 13$ se presentan las correlaciones desagregadas según el tipo de bebedor. En el caso de los abstemios en 30 días, las mujeres que bebían por motivos de estimulación reportaron tener más problemas asociados al consumo $(\mathrm{r}=.52)$. No se halló relación entre los motivos sociales y los problemas reportados.

En el caso de los bebedores ligeros, tanto hombres como mujeres que bebían por motivos de estimulación, presentaron problemas. Los hombres presentaron más problemas interpersonales que las mujeres. Tanto los hombres como las mujeres que bebían por estos motivos presentaron problemas de conducta. En cuanto a los motivos sociales, los hombres reportaron más problemas que las mujeres $y$, en ese senti-

Tabla № 13

Correlaciones entre los motivos para beber y los problemas asociados según género y tipología del bebedor

\begin{tabular}{|c|c|c|c|c|c|c|c|c|c|c|c|c|c|c|c|c|c|c|c|c|}
\hline & \multicolumn{20}{|c|}{ Tipo de bebedor } \\
\hline & \multicolumn{4}{|c|}{ Abstemio } & \multicolumn{4}{|c|}{ Ligero } & \multicolumn{4}{|c|}{ Moderado } & \multicolumn{4}{|c|}{ Intenso } & \multicolumn{4}{|c|}{ Problemático } \\
\hline & \multicolumn{2}{|c|}{ Mot1 } & \multicolumn{2}{|c|}{ Mot4 } & \multicolumn{2}{|c|}{ Mot1 } & \multicolumn{2}{|c|}{ Mot4 } & \multicolumn{2}{|c|}{ Mot1 } & \multicolumn{2}{|c|}{ Mot4 } & \multicolumn{2}{|c|}{ Mot1 } & \multicolumn{2}{|c|}{ Mot4 } & \multicolumn{2}{|c|}{ Mot1 } & \multicolumn{2}{|c|}{ Mot4 } \\
\hline & H & $\mathbf{M}$ & H & M & $\mathbf{H}$ & $\mathbf{M}$ & H & $\mathbf{M}$ & $\mathbf{H}$ & M & H & M & H & M & H & M & $\mathbf{H}$ & M & $\mathbf{H}$ & M \\
\hline Problema & .16 & $.52^{*}$ & .22 & 12 & $.66^{*}$ & $.40^{*}$ & $.55^{\star}$ & $.35^{*}$ & .13 & .25 & $.30^{*}$ & $.33^{*}$ & .29 & .27 & .10 & -.06 & $.52^{*}$ & .31 & $.35^{*}-$ & -.02 \\
\hline Inter. & .05 & .37 & .08 & -.01 & $.56^{\star}$ & $.37^{\star}$ & $.51^{*}$ & $.28^{*}$ & -.07 & .17 & .10 & .19 & .22 & -.19 & -.13 & -.23 & $.27^{\star}$ & .18 & $.33^{*}-$ & -.01 \\
\hline Conduc. & .00 & -.07 & .00 & -.21 & $.48^{*}$ & $.47^{*}$ & $.41^{*}$ & $.43^{*}$ & $.26^{*}$ & $.35^{\star}$ & $.37^{\star}$ & $.34^{*}$ & .05 & .25 & .07 & .08 & $.60^{*}$ & .07 & $.32^{*}$ & .01 \\
\hline
\end{tabular}

Nota. ${ }^{*} p<.05$. Mot1 = motivos de estimulación. Mot.4= motivos sociales. Inter. $=$ interpersonales. Conduc. $=$ conductuales. 
do, los hombres presentaron más problemas interpersonales que las mujeres; igualándose el reporte de problemas de conducta.

Los bebedores moderados que bebían por motivos de estimulación presentaron más problemas de conducta, siendo más fuerte en las mujeres que en los hombres. Mientras que los hombres y las mujeres que bebían por motivos sociales reportaron más problemas en general y, entre éstos, los de conducta.

Entre los bebedores intensos no se halló relación entre los motivos para beber y los problemas asociados. Los bebedores problemáticos varones que bebían por motivos de estimulación reportaron más problemas en general, así como también más problemas de relaciones interpersonales y de conducta, mientras que en las mujeres esta relación no se presentó. Los hombres que bebían por motivos sociales también presentaron problemas en general, interpersonales y de conducta.

La correlación parcial entre los estilos de afrontamiento y los problemas asociados, controlados por el género y la tipología, reportó que los otros estilos de afrontamiento se asociaban con los problemas en general. Se halló que las correlaciones simples, a nivel de escalas de afrontamiento y los problemas asociados, desaparecen al realizar las correlaciones parciales controladas por género y la tipología del bebedor.

Al hacer el análisis por cada tipo de bebedor y género, se encontró que los bebedores problemáticos que utilizaban otros estilos, tuvieron más problemas en general y esta asociación fue más fuerte en el caso de las mujeres. En los otros tipos de bebedores las correlaciones no fueron significativas.

\section{Discusión}

\section{Sobre los resultados descriptivos del consumo de bebidas alcohólicas}

La mayoría de la muestra está constituida por consumidores ligeros y moderados de acuerdo con el reporte de la cantidad de consumo de bebidas. Ello estaría ligado a la etapa de vida que atraviesan, en la cual la socialización, por los ritos de iniciación tanto en la adultez como en la vida universitaria, favorecen el consumo de alcohol (y/o drogas).

La muestra no constituye grupo de riesgo de consumo según los criterios planteados por The National Institute on Alcoholism and Alcohol Abuse (NIAA) (Schorling \& Buchsbaum, 1997) y constituye una excelente población para una adecuada labor preventiva cuyo diseño se vería favorecido por los datos complementarios de una muestra comparativa, con el fin de apreciar la evolución del consumo durante el período universitario.

Junto a un pequeño grupo de abstemios, se reporta -en el caso de la cerveza- un $14 \%$ de bebedores de riesgo (Schorling \& Buchsbaum, 1997), de 
quienes la literatura nos recuerda que tienden a presentar cuadros problemáticos en edades posteriores. Madrigal (1998) señala al respecto la importancia de una detección temprana de los bebedores en riesgo, pues intervenciones breves, como el consejo práctico y guías para cambios en la conducta de consumo, podrían ser suficientes para darles la opción de moderar su ingesta.

En relación con las bebidas consumidas se observa que son de diversos tipos (cerveza, licores fuertes y vino), lo que puede estar relacionado con el deseo de obtener experiencias nuevas, así como con el influjo de los medios de comunicación, a través de la modelación de personajes de cine, novela, líderes de opinión y publicidad. Esto nos lleva a recordar que la elevada producción de licor adulterado ejerce un impacto nocivo adicional sobre la salud de esta población.

La bebida alcohólica más consumida por estos jóvenes es la cerveza, resultado coincidente con el marcado aumento de su consumo entre 1960 y 1990 en muchos países de Sudamérica (OMS, 1994b) y en Perú (CEDRO, 1997). Conviene señalar que las políticas de publicidad restringen los horarios de los licores, mientras que la cerveza permanece irrestricta, lo que nos remite a la discusión de la conveniencia de la promoción de la cerveza para reducir el impacto nocivo de los licores.

En este estudio, al igual que en otro realizado en una muestra similar en
1993, se halló que el consumo de alcohol se acompaña principalmente de cigarrillos de tabaco, resultado coincidente con estudios hechos por CEDRO en 1997. Se ha apreciado una asociación del consumo de alcohol a otras sustancias psicoactivas ilegales, principalmente marihuana; la literatura nos recuerda que la asociación podría derivar hacia una intensificación progresiva del consumo y una secuencialidad hacia consumos de mayor nocividad y riesgo (Thorlindsson \& Vilhalmsson, 1991; Villanueva, 1989b).

Con relación al género, los varones consumen más alcohol que las mujeres, casi el doble, siendo un reporte coincidente con la literatura reciente (NIAA, 1997b; CEDRO, 1997). Convendría precisar si los factores que inciden en este resultado están más relacionados con los modelos publicitarios orientados a varones o con mayores niveles de estrés en éstos. Si bien las mujeres reportan menor consumo, debemos recordar que se encuentran orgánicamente más expuestas a los efectos nocivos del alcohol.

Se ha propuesto la tipología del bebedor de Windle, que ha permitido una mejor aproximación a la muestra proporcionando criterios efectivos de diferenciación y un manejo adecuado de los resultados. Así, una diferenciación de los porcentajes de la frecuencia vinculada al género es evidente entre los abstemios, consumidores ligeros y los problemáticos, mientras que en los mo- 
derados e intensos disminuye notoriamente. De igual forma, ha permitido una mejor caracterización en función a otras variables de nuestros estudio, como las formas del consumo, los estilos de afrontamiento al estrés y las motivaciones para beber.

Un hallazgo que llama la atención es que el $22 \%$ se ubique en la categoría bebedor problemático, cuyo consumo puede estar lindando con el abuso, dato que resulta interesante si "se considera que las cifras de dependencia, aún cuando incompletas e indirectas, dan cuenta de guarismos que oscilan entre el $9 \%$ y el $25 \%$ en la población peruana" (Saavedra, 1997). Asimismo, este autor plantea el interés por las dependencias en la adolescencia que ha adquirido notorio desarrollo en la última década, a pesar de que los criterios de abuso y dependencia no suelen plantearse con tanta frecuencia en esta etapa de la vida. Esto plantea toda una línea de investigación a realizar.

Con respecto a los estilos de afrontamiento, se observa que los orientados a la emoción tienen predominio entre los no consumidores, y la mayor frecuencia corresponde a acudir a la religión y a la reinterpretación positiva. Sin embargo, también son utilizados entre los consumidores. En efecto, aun cuando los consumidores presentan el recurso a todos los estilos de afrontamiento, reportan mayor frecuencia en los estilos orientados a la emoción, en especial las mujeres, lo que coincide con el hallaz- go de Halstead, Bennett \& Cunningham (1993), de que los jóvenes tienden a utilizar múltiples estrategias de afrontamiento cuando se enfrentan a una situación estresante.

Al margen de las características sociales que refieren a un mayor uso de los estilos orientados a la emoción, estos resultados nos recuerdan la importancia de orientar el trabajo preventivo al fomento de un mayor recurso en los estilos orientados al problema, que han sido planteados como los de mayor eficacia para el manejo de situaciones estresantes.

El consumo de estos jóvenes se hace en grupo, fiestas y con amigos. Esto coincide con la literatura que reporta una mayor incidencia de motivaciones sociales y de estimulación. A pesar de ello, la muestra no reporta ningún motivo para beber especialmente destacable, lo que tal vez nos refiera a los criterios individuales de discriminación entre los diferentes motivos, vinculados a cierta susceptibilidad propia de la edad, como son el aceptar la presión de la influencia del medio o el recurso para afrontar situaciones, etc.

Estos hallazgos se aproximan a los de CEDRO en 1997, en donde, en segundo lugar se mencionan las razones asociadas a situaciones placenteras, consumo recreacional en fiestas y grupos. Nuestros hallazgos también corroboraron los estudios en donde se reporta que los motivos sociales están más relacionados con el uso de alcohol más ligero, infrecuente y no problemático y 
en situaciones sociales. Asimismo, los motivos de estimulación están vinculados con el consumo en lugares en donde es tolerado y, además, estaría relacionado con la búsqueda de emociones positivas, que es parte del comportamiento social de los jóvenes. Además, Milhorn (1990) señaló que la razón predominante por la que los adolescentes recurren en general a las drogas, es que éstas los hacen sentirse bien y no experimentan consecuencias adversas.

Respecto de los problemas relacionados al consumo, la mayor frecuencia se encuentra entre los referidos a relaciones interpersonales: tener discusiones con los padres o con el/la enamorada/o por la bebida, así como pensar en reducir la cantidad y arrepentirse de las cosas hechas bajo los efectos de la bebida. Casi no se reportan problemas de conducta o en el área académica.

\section{Correlaciones entre la cantidad consumida de alcohol, los motivos para beber, los problemas relaciona- dos y los estilos de afrontamiento}

Los motivos de conformidad, por sí solos y aisladamente, no presentan relación con la cantidad consumida, lo que coincide con la literatura según la cual los motivos internos están vinculados a un consumo más elevado, lo que se expresa en la muestra con una correlación de los motivos de estimulación y de afrontamiento, con la cantidad de consumo. Así como también los motivos sociales se asocian con la can- tidad y frecuencia de consumo (Cooper, 1994).

Estos tres motivos se correlacionan también con la tipología del bebedor: los tipos más intensos responden a mayores niveles de estimulación en estos tres motivos, lo que recuerda la importancia del factor cantidad de consumo en la caracterización ordinal de la tipología propuesta, resultado convergente respecto de ésta.

Al correlacionar la cantidad consumida con los estilos de afrontamiento no se encuentra asociación con los estilos orientados al problema, lo que es contrario a la literatura existente, que plantea que los afrontamientos activos son predictores de la disminución del riesgo de abuso. Por el contrario, a nivel de escalas, se encuentra que la búsqueda de apoyo social instrumental sí correlaciona con la cantidad en varones, lo que abre interrogantes a investigaciones posteriores. En cambio, las estrategias centradas en enfocar y liberar emociones sí correlacionan con la cantidad de consumo, lo que coincide con la literatura referida a los estilos evitativos que se asocian a consumos más elevados (Wills \& Hirky, 1996).

Si bien los estilos orientados a la emoción no correlacionan con la cantidad, a nivel de escalas se encuentra que los que utilizan la aceptación como forma de afrontamiento consumen más, en especial los varones, mientras que los que utilizan la religión consumen menos, en especial las mujeres. 
La muestra asocia la cantidad de consumo a la presencia de problemas, lo que nos remite a la literatura general sobre el consumo y permite comprobar la confiabilidad de los criterios usados para la conformación de la tipología, que a mayor consumo asocia presencia progresiva de problemas.

Los motivos sociales y de estimulación son los que más se han relacionado con los problemas en cada tipo de bebedor, en los cuales el género y la tipología del bebedor tienen un efecto importante. Esto permite plantear que los problemas no son exclusivos del tipo de bebedor problemático, sino que se presentan en todos los jóvenes de la muestra. En relación con los motivos de estimulación, el alcohol puede incrementar el afecto positivo, disminuir, el aburrimiento y el humor disfórico. Se confirma lo propuesto por Woody et al. (1992), quienes plantearon que estas razones han sido reportadas en el uso inicial y repetitivo de alcohol, en personas que abusaron del alcohol y que luego estuvieron bajo tratamiento.

En relación con los motivos sociales, los estudiantes estarían bebiendo con la finalidad de obtener un resultado positivo del medio o recompensas sociales. En ese sentido, Kellin et al. en 1996 (Bandura, 1997), en un estudio prospectivo sobre el inicio y mantenimiento del consumo de alcohol en adolescentes, plantearon que el principal predictor del inicio, tanto en chicos como en chicas, fue la expectativa de que el alcohol elevaba o mejoraba el funcionamiento social. A esto se une que la práctica sociocultural ejerce una fuerte influencia sobre los patrones de consumo. El moldeamiento social de la conducta de beber es un poderoso instigador y regulador del consumo de alcohol (Bandura, 1997).

Llama la atención que en los bebedores intensos, la relación entre los motivos y los problemas desaparezca; quizás esto se deba a que al estar en un estadio inmediato anterior al consumo problemático, estas variables ya no sean importantes porque su efecto se dio en estadios previos.

Por último, los bebedores problema varones que beben para estimularse reportan dificultades sobre todo de conducta e interpersonales. Este dato debe ser tomado con cuidado pues en la construcción de la tipología, la definición de este tipo de bebedor incluye el reporte de cinco problemas. Estos bebedores que lo hacen por motivos sociales tienen problemas en general y especialmente interpersonales y de conducta. Es interesante que la relación no se dé para las mujeres, lo que podría explicarse por las diferencias en el consumo en relación con el género, en las cuales los hombres necesitan más alcohol para expresarse y tienen más oportunidad de hacerlo. En ese sentido, es preocupante que, debido a que los jóvenes raramente beben solos, se ven frecuentemente implicados en actos de violencia, altercados públicos, conflic- 
tos con los agentes del orden, accidentes de tránsito, etc.

La tipología del bebedor y el género influyen en la relación entre los estilos de afrontamiento y los problemas relacionados con el consumo, siendo relevante señalar que, en general, los jóvenes que tienen un consumo problemático y que utilizan otros estilos de afrontamiento, reportan más problemas.

En relación con los estilos de afrontamiento y su asociación con la intensidad del consumo - entendida como la gradación en el consumo dada por la tipología del bebedor- son las mujeres con un consumo más problemático las que tienden a utilizar más los otros estilos de afrontamiento, como enfocar y liberar emociones y desentendimiento mental. Esto indicaría que en el intento de solucionar dificultades utilizan formas de afrontamiento que no son tan adaptativas y que se asocian precisamente con un consumo riesgoso.

Los jóvenes aquí estudiados, quienes tienen un consumo más problemático, tienden más a enfocar y liberar emociones, lo cual podría ser doblemente peligroso, debido a que mediante esta estrategia estarían focalizándose en las consecuencias emocionales desagradables que sienten, expresándolas abiertamente. Asimismo, tienden a acudir menos a la religión como una forma de afrontamiento. Así también, los hombres que consumen más no utilizan la postergación del afrontamiento, que consiste en esperar hasta que se presen- te la oportunidad apropiada para actuar sobre el problema, evitando hacer algo prematuramente.

Todo esto nos lleva a preguntarnos si estos jóvenes universitarios no poseen o no encuentran otras respuestas de afrontamiento disponibles y si es necesario diseñar programas sobre el manejo del estrés. Esto último se torna crucial porque justamente son los jóvenes que tienen un consumo riesgoso los que generalmente no cuentan con un repertorio adecuado de conductas que les permita evitar buscar en el alcohol una forma de hallar soluciones a sus problemas. A pesar de que se sigue considerando a la educación como el mecanismo más efectivo de movilidad social y como sinónimo de mejora de ingresos y condiciones de vida, es innegable que no garantiza lo que se espera de ella. En esta etapa recesiva de la economía, es similar el panorama en lo que respecta a las oportunidades laborales (Cisneros y Llona, 1997). Todo ello implica que los jóvenes, muchas veces, se tienen que enfrentar con un medio cada vez más complejo y deben afrontar situaciones para las cuales no están necesariamente preparados. Por esto consideramos que el espacio universitario podría proporcionarles no sólo habilidades académicas, sino también habilidades para la vida (solución de problemas, habilidades sociales $\mathrm{y}$ autorregulación de las emociones).

A partir de este estudio pensamos, en cuanto al área de investigación, que 
existe la necesidad de continuar desarrollando estudios con población universitaria procedente de instituciones privadas y estatales, que nos permita ampliar nuestro marco de comprensión de los fenómenos psicológicos que se presentan asociados al consumo de bebidas alcohólicas, en este estadio de la vida. Además, esto podría permitir el desarrollo de programas específicos de prevención, en la medida en que se identifiquen grupos diferenciados en el consumo de alcohol.

Se resalta la importancia del desarrollo de estudios longitudinales, los que posiblemente pueden ser llevados a cabo en universidades privadas y estatales; así como también en centros de estudios no universitarios.

Sería conveniente para futuras investigaciones, tomar en cuenta variables de personalidad, como las expectativas asociadas al consumo de bebidas alcohólicas. Esto debido a que el impacto combinado de las influencias sociales y la eficacia percibida en el uso temprano del alcohol, cigarrillos y marihuana está documentada en una investigación longitudinal de Ellickson \& Hays en 1991 (Bandura, 1997).

Consideramos que a partir de los hallazgos podemos sugerir que las estrategias de prevención de la drogadicción, incluido el alcoholismo, se centre no sólo en la información sobre los efectos que provoca el alcohol en el organismo y sus consecuencias para la salud, sino también en el desarrollo de la competencia individual basándose en el entrenamiento de habilidades (tomar decisiones, comunicación interpersonal), y del autocontrol emocional. Especialmente porque las motivaciones más saltantes para beber son las de afrontamiento.

\section{REFERENCIAS}

American Psychiatric Association. (1994). Diagnostic and statistical manual of mental disorders (4a. ed.). Washington, DC: APA.

Annis, H. \& Davis, Ch. (1985). Assesment of expectancies. En G. Marlatt, J. Gordon (Eds.), Relapse prevention (pp. 84111). Nueva York: The Guilford Press.

Bandura, A. (1997). Self-efficacy. The excercise of control. Nueva York: W. H. Freeman.

Barnes, G. (1990). Impact of the familiy on adolescent drinking patterns. En R. Collins, K. Leonard \& J. Sarles (Eds.), Alcohol and the family: Research and clinical perspectives (pp. 137-161). Nueva York: Guilford Press.

Beck, J. (1995). Cognitive therapy. Basics and beyond. Nueva York: Guilford Press.

Billings, A. \& Moos, R. (1982). Stressful life events and symptoms: a longitudinal model. Health Psychology, 1, 99117.

Blechman, E. \& Wills, T. (1992). Process measures in intervention for drug-abusing women: from coping to competence. En M. Kilbey \& K. Asghar (Eds.), Methodological issues in epidemiological, prevention, and treatment research on drug-exposed women 
and their children (pp. 314-343). Rockville, MD: National Institute on Drug Abuse.

Carver, C., Scheier, M. \& Weintraub, J. (1989). Assesing coping strategies: a theoretically based approach. Journal of Personality and Social Psychology, 56, 267-283.

Casuso, L. (1996). Adaptación de la prueba COPE sobre estilos de afrontamiento en un grupo de estudiantes universitarios de Lima. Tesis de licenciatura no publicada, Pontificia Universidad Católica del Perú, Lima.

CEDRO (1993). Opiniones sobre drogas en el Perú población urbana. Monografía de Investigación $\mathrm{N}^{\circ}$ 8. Lima: CEDRO.

CEDRO (1997). Epidemiología de drogas en la población peruana-1995 (Encuesta en hogares). Monografía de Investigación $\mathrm{N}^{\mathrm{o}}$ 15. Lima: CEDRO.

Christiansen, B., Goldman, M. \& Inn, A. (1982). Development of alcohol-related expectancies in adolescents: Separating pharmacological from social-learning influences. Journal of Consulting and Clinical Psychology, 50, 336-344.

Cisneros, L. \& Llona, M. (1997). Por una zona franca para los jóvenes. Lima: DESCO.

Cohen, J. (1988). Statistical power analysis for the behavioral sciences. Nueva Jersey: Lawrence Erlbaum.

Cooper, L., Russell, M., Skinner, J., Frone, M. \& Mudar, P. (1992). Stress and alcohol use: moderating effects of gender, coping, and alcohol expectancies. Journal of Abnormal Psychology, 101(1), 139-152.
Cox, M. \& Klinger, E. (1988). A motivational model of alcohol use. Journal of Abnormal Psychology, 97(2), 168-180.

Cox, M. \& Klinger, E. (1990). Incentive motivation, affective change and alcohol use: A model. En M. Cox (Ed.), Why people drink (pp. 291-311). Nueva York: Gardner Press.

Denoff, M. (1988). An integrated analysis of the contribution made by irrational beliefs and parental interaction to adolescent drug abuse. The International Journal of the Addictions, 23(7), 655669.

Endler, N. \& Parker, J. (1990). Multidimensional assesment of coping: A critical evaluation. Journal of Personality and Social Psychology, 58, 844-854.

Evans, M. \& Jo Dunn, N. (1995). Alcohol expectancies, coping responses and self-efficacy judgments: a replication and extension of Cooper et al., 1988 study in a college sample. Journal of Studies on Alcohol, 56, 186-193.

Ferrando, D. (1990). Uso de drogas en las ciudades del Perú. Monografía de Investigación $\mathrm{N}^{\circ}$ 5. Lima: CEDRO.

Ferrando, D. (1991). Los jóvenes en el Perú. Opciones, actitudes y valores. Lima: CEDRO.

Ferrando, D. (1992). Conocimiento y uso de drogas en los colegios secundarios. Encuesta nacional 1992. Lima: Ministerio de Educación

Finney, J. \& Moos, R. (1992). The longterm course of treated alcoholism: predictors and correlates of 10-year functioning and mortality. Journal of Studies of Alcohol, 53, 142, 153.

Folkman, S. \& Lazarus, R. (1980). An analysis of coping in middle-aged commu- 
nity sample. Journal of Health and Social Behavior, 21, 129-239.

Halstead, M., Bennett, S. \& Cunningham, W. (1993). Measuring coping in adolescents: An application of the ways of coping checklist. Journal of Clinical Child Psychology, 22, 337-344.

Huamán, J., Tueros, M. \& Villanueva, M. (1983). Aspectos psicosociales relacionados con el uso de drogas en adolescentes de Lima Metropolitana. Manuscrito no publicado. Pontificia Universidad Católica del Perú, Lima.

Jessor, R. \& Jessor, S. (1977). Problem behavior and psychosocial development: a longitudinal study of youth. Nueva York: Academic Press.

Jutkowitz, J., et al. (1987). Uso y abuso de drogas en el Perú. Monografía de Investigación $N^{\circ}$ 1. Lima: CEDRO.

Kline, P. (1993). The handbook of psychological testing. Londres: Boutledge.

Klinger, E. (1975). Consequences of commitment to and disengagement from incentives. Psychological Review, 82, 125 .

Klinger, E. (1977). Meaning and void: Inner experience and the incentives in peoples's lives. Minneapolis: University of Minnesota Press.

Lazarus, R. (1991). Progress on a cognitive-motivational-relational theory of emotion. American Psychologist, 46, (8), 819-834.

Lazarus, R. \& Folkman, S. (1984). Stress, appraisal and coping. Nueva York: Springer Publishing.

Lazarus, R. \& Folkman, S. (1986). Estrés y procesos cognitivos. Barcelona: Martínez Roca.
León, F., Ugarriza, G. \& Villanueva, M. (1989). La iniciación y el uso de sustancias psicoactivas: un análisis de datos en una encuesta nacional de colegios. Lima: Ministerio de Educación, Convenio Perú-AID.

Madrigal, E. (1998). Detección y atención tempranas. "El bebedor problema". Médico Interamericano, 17(8), 418422.

Marlatt, G. (1988). Situational determinants of relapse and skill-training interventions. En D. Donnovan \& G. Marlatt. (Eds.), Assessment of addictive behavior (pp. 71-98). Nueva York: The Guilford Press.

Marlatt, G. \& Rohsenow, D. (1980). Cognitive processes in alcohol use: Expectancy and the balanced placebo design. En N. Mello (Ed.), Advances in substance abuse: Behavioral and biological research (pp.159-199). Greenwich, $\mathrm{Ct}$ : JAI Press.

Milhorn, T. (1990). Chemical dependence. Diagnosis, treatment, and prevention. Nueva York: Springer-Verlag.

O'Conell, D. \& Patterson, H. (1989). A survey of current college alcohol abuse programs, attitudes and training needs. Journal of Alcohol and Drug Education, 43(2), 61-69.

Organización Mundial de la Salud (1994a). Léxico de los términos del alcohol y drogas. Ginebra.

Organización Mundial de la Salud (1994b). Alcohol y salud pública. Barcelona: J. R. Prous.

Pandina, R, Johnson V. \& Labovie, E. (1992). Affectivity: a central mechanism in the development of drug dependence. En M. Glantz y R. Pickens 
(Eds.), Vulnerability to drug abuse (pp. 179-209). Washington, DC: American Psychological Association.

Rohde, P., Lewinsohn, P., Tilson, M. \& Seeley, J. (1990). Dimensionality of coping and its relation to depression. Journal of Personality and Social Psychology, 58, 499-511.

Saavedra, A. (1997). Comorbilidad en el alcoholismo. Serie de Monografías de Investigación $\mathrm{N}^{\circ}$ 5. Lima: INSM.

Schorling, J. \& Buschsbaum, D. (1997). Screening for alcohol and drug abuse. The Medical Clinics of North America, 81(4), 845-865.

Simons, R., Conger, R. \& Whitbeck, L. (1988). A multistage social learning model of the influences of family and peers upon adolescentes substance abuse. The Journal of Drug Issues, 18(3), 293-315.

Sotomayor, J. (1976). El problema de las drogas en un grupo de estudiantes de secundaria de Lima. En R. Alarcón, J. Infante, C. Ponce. \& A. Bibolini. (Eds.), La investigación psicológica en el Perú. Lima: Sociedad Peruana de Psicología.

Stone, A., Greenberg, M., Kennedy-Moore, E. \& Newman, M. (1991). Self-report situation-specific coping questionnaires: What are they measuring? Journal of Personality and Social Psychology, 61, 648-658.

Thorlindsson, T. \& Vilhjalmsson, R. (1991). Factors related to cigarrete smoking and alcohol use among adolescents. Adolescence, 26(102), 399418.

Villanueva, M. (1989a). El conocimiento de los adolescentes sobre drogas. Lima: Ministerio de Educación.
Villanueva, M. (1989b). Factores psicológicos asociados a la iniciación y habituación. En F. León \& R. Castro de la Mata (Eds.), Pasta básica de cocaína. Un estudio multidisciplinario (pp. 167209). Lima: CEDRO.

Villanueva, M. (1996). Middle class adolescents in Lima. A description of the psychosocial aspects and the drug use incidence. Tesis doctoral no publicada, Universidad Católica de Nimega, Holanda.

Windle, M. (1996). An alcohol involvement typology for adolescents: Convergent validity and longitudinal stability. Journal of Studies on Alcohol, 57, 627637.

Windle, M. \& Windle, R. (1996). Coping strategies, drinking motives, and stressful life events among middle adolescents: Associations with emotional and behavioral problems and with academic functioning. Journal of Abnormal Psychology, 105(4), 551-560.

Wills, T. \& Hirky, E. (1996). Coping and substance abuse: A theoretical model and review of the evidence. En $\mathrm{M}$. Zeinder \& N. Endler (Eds.), Handbook of coping. Toronto: John Wiley.

Woody, G., Urschel, H. \& Alterman, A. (1992). The many path to drug dependence. En. M. Glantz \& R. Pickens (Eds.), Vulnerability to drug abuse (pp. 491-507). Washington DC: American Psychological Association. 